\title{
Multiscale InSAR Time Series (MInTS) analysis of surface deformation
}

\author{
E. A. Hetland, ${ }^{1}$ P. Musé, ${ }^{2}$ M. Simons, ${ }^{3}$ Y. N. Lin, ${ }^{3}$ P. S. Agram, ${ }^{3}$ and C. J. DiCaprio ${ }^{3,4}$
}

Received 29 July 2011; revised 12 December 2011; accepted 14 December 2011; published 18 February 2012.

[1] We present a new approach to extracting spatially and temporally continuous ground deformation fields from interferometric synthetic aperture radar (InSAR) data. We focus on unwrapped interferograms from a single viewing geometry, estimating ground deformation along the line-of-sight. Our approach is based on a wavelet decomposition in space and a general parametrization in time. We refer to this approach as MInTS (Multiscale InSAR Time Series). The wavelet decomposition efficiently deals with commonly seen spatial covariances in repeat-pass InSAR measurements, since the coefficients of the wavelets are essentially spatially uncorrelated. Our time-dependent parametrization is capable of capturing both recognized and unrecognized processes, and is not arbitrarily tied to the times of the SAR acquisitions. We estimate deformation in the wavelet-domain, using a cross-validated, regularized least squares inversion. We include a model-resolution-based regularization, in order to more heavily damp the model during periods of sparse SAR acquisitions, compared to during times of dense acquisitions. To illustrate the application of MInTS, we consider a catalog of 92 ERS and Envisat interferograms, spanning 16 years, in the Long Valley caldera, CA, region. MInTS analysis captures the ground deformation with high spatial density over the Long Valley region.

Citation: Hetland, E. A., P. Musé, M. Simons, Y. N. Lin, P. S. Agram, and C. J. DiCaprio (2012), Multiscale InSAR Time Series (MInTS) analysis of surface deformation, J. Geophys. Res., 117, B02404, doi:10.1029/2011JB008731.

\section{Introduction}

[2] Geodetic imaging aims to discover new crustal deformation processes, to monitor known sources of deformation, and to estimate the values and uncertainties of the parameters controlling these processes. Here, we focus on the use of repeat pass satellite interferometric synthetic aperture radar (InSAR) data. (For a review of InSAR techniques, we refer the reader to Simons and Rosen [2007] and references therein.) For many important geophysical targets, we already have deep archives of radar images from a given satellite and viewing geometry. More importantly, future radar missions should provide frequent image acquisitions with high interferometric correlation. In response to the increase in temporal density of radar acquisitions, we present a new approach to exploit InSAR archives to determine the spatiotemporal evolution of surface deformation.

[3] The simplest approach to utilizing multiple interferograms is to average them (often referred to as "stacking", although in this paper we do not use the term "stack" to be

\footnotetext{
${ }^{1}$ Department of Earth and Environmental Sciences, University of Michigan, Ann Arbor, Michigan, USA.

${ }^{2}$ Department of Signal and Image Processing, IIE, Facultad de Ingenieria, Universidad de la Republica, Montevideo, Uruguay.

${ }^{3}$ Seismological Laboratory, California Institute of Technology, Pasadena, California, USA.

${ }^{4}$ Now at ExxonMobil Upstream Research Co., Houston, Texas, USA.

Copyright 2012 by the American Geophysical Union. 0148-0227/12/2011JB008731
}

synonymous with "average"). If the primary geophysical target is a single event that occurred quickly (i.e., anything taking less than the image acquisition interval) or is a gradual process occurring at constant rate, averaging is commonly used to increase the signal-to-noise ratio [e.g., Peltzer et al., 2001; Lyons and Sandwell, 2003; Gourmelen and Amelung, 2005; Pritchard and Simons, 2006]. For example, averaging reduces the effects due to tropospheric delays, as these effects are typically uncorrelated on timescales of more than a day [e.g., Hanssen, 2001; Emardson et al., 2003]. Averaging images can also reduce computational burden in parameter estimation schemes by reducing the amount of observations [e.g., Pritchard and Simons, 2006]. In the case of a single rapid event, the displacements are averaged, whereas for a constant rate process, it is common to average the velocities. We note that by averaging a set of interferograms, one is implicitly assuming a functional form for the deformation field (a step or a linear function) and simply estimating the appropriate constants [Simons and Rosen, 2007].

[4] Over the last decade, the community has made considerable progress in estimating time-dependent deformation from InSAR data. Approaches include Permanent or Persistent Scatterer (PS) techniques [e.g., Ferretti et al., 2000, 2001; Colesanti et al., 2003; Wegmüller, 2003; Hooper et al., 2004; Bürgmann et al., 2006] as well as interferogram time series techniques [e.g., Lundgren et al., 2001, 2009; Berardino et al., 2002; Schmidt and Bürgmann, 2003; Lanari et al., 2004; Hooper, 2008]. Most of these techniques exploit long time series of over a decade of SAR observations. PS techniques are restricted to using only temporally coherent 
point scatterers, and require a parametrization of the time dependent deformation. In contrast, other time series techniques use all available pixels in all scene combinations where the interferometric baseline is well below a critical baseline [Lundgren et al., 2001; Berardino et al., 2002; Schmidt and Bürgmann, 2003; Lanari et al., 2004]. As a result, in the latter techniques one can expand the area of usable pixels, and for well-correlated areas, potentially the time-dependent deformation of every pixel could be determined. For each usable pixel, interferometric pairs yield phase differences over available time intervals in the time series, with the shortest possible sampling interval being the satellite repeat period. In contrast to PS techniques, the phase difference measurements at a given pixel in all combinations of radar images are inverted to determine the time-dependent ground deformation. Typically, a piecewise linear timedependence is sought, with the most common version of this latter technique being the Small Baseline Subset (SBAS) method [e.g., Lundgren et al., 2001; Berardino et al., 2002; Doubre and Peltzer, 2007]. Recently, Hooper [2008] proposed a hybrid approach to estimating time-dependent ground deformation, utilizing both PS and SBAS methodologies.

[5] While providing a solid foundation, these existing approaches have several important shortcomings. Principally, current methods are applied on a pixel-by-pixel basis, with pixel-to-pixel connection made by assuming a single master reference image. Pixel-by-pixel methods ignore the known spatial covariances in the observations, as well as expectations for "reasonable" behavior even in regions of low interferometric correlation. Current methods also use ad-hoc strategies to deal with interferograms containing regions of low interferometric correlation, or to deal with sets of interferograms that are not connected to other interferograms by common image acquisitions.

[6] We propose a multiscale InSAR time series (MInTS) approach to determine the spatiotemporal ground deformation from a catalog of interferograms of a region. For brevity, we refer to a catalog of interferograms as a "stack" throughout this paper. In our approach, we use a wavelet decomposition in space and a general parametrization in time. Spatial wavelet decompositions have been effectively applied to InSAR data in order to provide better estimates of orbital errors [Shirzaei and Walter, 2011]. Additionally, although not using wavelet based filters, Lin et al. [2010] used a multiscale decompositions in order to provide a more robust estimation of topographically correlated tropospheric path delays. The spatial wavelet decomposition we use in MInTS is chosen in order to efficiently deal with the spatial covariances in the InSAR phase difference measurements and to provide an efficient method for interpolating across regions of low interferometric correlation.

[7] Our approach to the temporal parametrization is highly influenced by common approaches used for post-processing continuous GPS data to extract velocities, co-seismic offsets, etc (e.g., QOCA and GLOBK) [Herring et al., 1990; Dong et al., 1998, 2002]. Determination of time-dependent ground deformation using InSAR data substantially differs from GPS time series techniques, as InSAR has a much higher density of samples in space, but much lower density of samples in time. Moreover, InSAR measures differences in ground position over time spans between repeat orbits, whereas GPS measures position through time relative to some reference. In this paper, we only focus on unwrapped interferograms from a single viewing geometry, thereby only resolving ground deformation along the satellite line-of-sight (LOS). In the following, we describe the theory and methodology of MInTS, we illustrate the application of MInTS to an example InSAR stack from Long Valley caldera, CA, and we discuss some future directions of MInTS development.

\section{Methods}

[8] In this section, we outline the methods behind MInTS (refer to Figure 1 for a conceptual outline of the stages involved in the MInTS analysis). We first describe our temporal parametrization of the time dependent deformation, how this parametrization applies to interferometric measurements, and illustrate this time parametrization using a synthetic single pixel example. We then describe the full data covariances of a stack of interferograms, the multiscale spatial decomposition using 2D wavelets, and the application of the discrete wavelet transform to interferograms. Finally, we describe the inversion method used in MInTS and the reconstruction of the inferred spatiotemporal deformation field. The entire MInTS analysis has been implemented as a Matlab (The Mathworks, Inc.) toolbox freely available from the authors.

\subsection{Parametrization of Time-Dependent Deformation}

[9] We construct a general parametrization of timedependent ground deformation, capable of parameterizing known processes, as well as capturing unknown processes. For a given location, $\Omega=\Omega(x, y)$, and time, $t$, we represent the projection of the $3 \mathrm{D}$ surface deformation into the satellite line-of-sight (LOS) as

$$
\rho(\Omega, t)=\rho_{o}(\Omega)+F_{\rho}(\Omega, t),
$$

where, $\rho_{o}(\Omega)$ is a location specific constant, and $F_{\rho}(\Omega, t)$ can be any time-dependent function. For now we consider $F_{\rho}(\Omega, t)$, in its most complete form, to be

$$
\begin{aligned}
F_{\rho}(\Omega, t)= & v_{\rho}(\Omega) t+ \\
& \sum_{i \in \mathcal{I}_{\Delta}} \Delta_{i}(\Omega) \mathcal{H}\left(t-T_{i}\right)+ \\
& \sum_{i \in \mathcal{I}_{L}} \alpha_{i}^{L}(\Omega) \mathcal{H}\left(t-T_{i}^{L}\right) \ln \left(1+\frac{t}{\tau_{i}^{L}}\right) \\
& \sum_{i \in \mathcal{I}_{E}} \alpha_{i}^{E}(\Omega) \mathcal{H}\left(t-T_{i}^{E}\right)\left[1-e^{\left.\frac{-t}{\tau_{i}^{E}}\right]+}\right. \\
& \sum_{i \in \mathcal{I}_{P}}\left[s_{i}(\Omega) \sin \left(\omega_{i} t\right)+c_{i}(\Omega) \cos \left(\omega_{i} t\right)\right]+ \\
& \sum_{i \in \mathcal{I}_{U B}} \kappa_{i}(\Omega) B_{n}\left(t-t_{i}^{b}\right)+ \\
& \sum_{i \in \mathcal{I}_{I B}} \kappa_{i}^{\prime}(\Omega) B_{n}^{\int}\left(t-t_{i}^{b}\right)+ \\
& \sum_{i \in \mathcal{I}_{N B}} \kappa_{i}^{\prime \prime}(\Omega) B_{n i}\left(t-t_{i}^{\#}\right),
\end{aligned}
$$




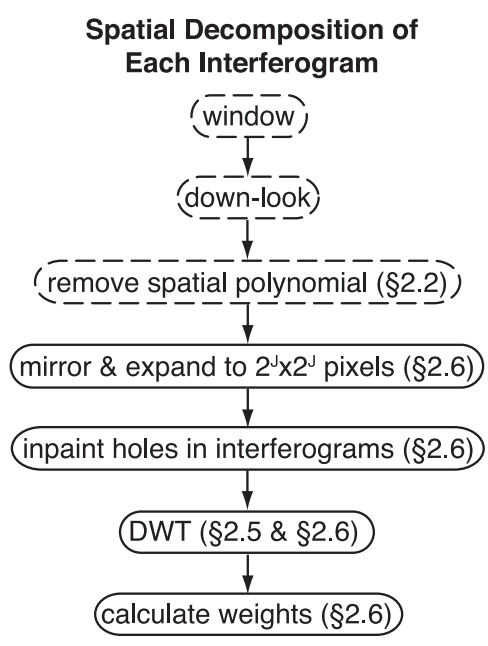

Spatial Decomposition of

(window)

(awn-look

remove spatial polynomial $(\S 2.2)$

mirror \& expand to $2^{\lrcorner} \times 2^{J}$ pixels $(\S 2.6)$
Estimation of Time-Dependent Deformation

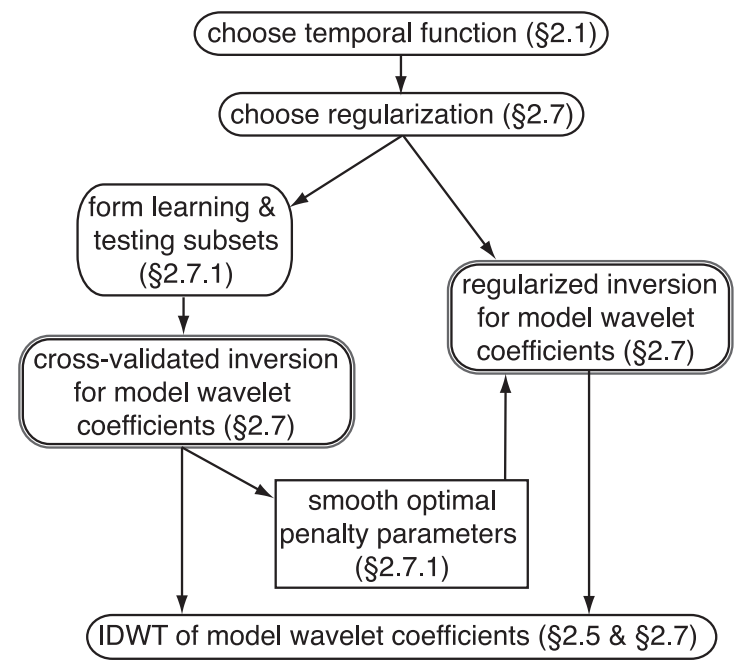

Figure 1. Outline of MInTS analysis. (left) Stages of processing each interferogram for MInTS analysis: dashed ovals are optional stages, solid ovals are required. (right) Stages in the inversion of the InSAR stack in the wavelet domain: double lined ovals indicate stages repeated independently at each wavelet location and scale, single lined ovals are stages common to all locations and scales. Section number refers to explanation of the stage in the main text.

where $\mathcal{H}\left(t-T_{i}\right)$ is a Heavyside function centered at $T_{i}$, $B_{n}\left(t-t_{i}^{b}\right)$ are B-splines of order $n$ centered on the uniformly spaced knots $t_{i}^{b}, B_{n}^{\int}\left(t-t_{i}^{b}\right)=\int B_{n}\left(t^{\prime}-t_{i}^{b}\right) d t^{\prime}$, and $B_{n i}\left(t-t_{i}^{\#}\right)$ are B-splines of order $n$ located at the non-uniformly spaced knots $t_{i}^{\#}$. We define $\mathcal{I}_{i}$ to be a set of indices of a collection of functional forms. Note that when $\mathcal{I}_{i}$ is an empty set, the $i$ 'th function is not included in the parametrization of the time-dependent deformation. The spatially dependent coefficients $\Delta_{i}, \alpha_{i}^{L}, \alpha_{i}^{E}, s_{i}, c_{i}, \kappa_{i}, \kappa_{i}^{\prime}$, and $\kappa_{i}^{\prime \prime}$ are unknown parameters that will be estimated from the InSAR stack.

[10] The first term in $F_{\rho}(\Omega, t)$ is a linear rate of deformation. The second term describes offsets at specific times, such as those due to earthquakes. This term can also capture noninstantaneous offsets whose time span is below the temporal resolution of the InSAR stack. The second two terms, (4) and (5), are often used in geodetic studies to represent postseismic deformation [e.g., Savage et al., 2003, 2005], although we note that the time constants $\alpha_{i}^{L}$ and $\alpha_{i}^{E}$ in terms (4) and (5), respectively, are typically taken a priori, and alone they have no direct physical meaning [e.g., Hetland and Hager, 2006]. The functions in terms (4) and (5) are one-sided, and are characterized by sharp onsets, and steady decay (Figure 2). Term (6) describes periodically repeating deformation. When $\mathcal{I}_{P}=\{1,2\}, \omega_{1}=2 \pi / \mathrm{yr}$, and $\omega_{2}=4 \pi / \mathrm{yr}$, term (6) is the common approximation of ground deformation due to annual and bi-annual seasonal variations of groundwater storage [e.g., Dong et al., 2002]. This simple sinusoidal representation of seasonal ground deformation is only an approximation, and actual ground deformation due to seasonal variations in groundwater are more complicated [e.g., Bennett, 2008]. More complicated descriptions of the seasonal terms can be incorporated into $F_{\rho}$.

[11] The final three terms in $F_{\rho}(\Omega, t)$, terms (7)-(9), do not carry any assumption of what the underlying component of the deformation signal is due to. Instead, we use these basis functions to describe signals that are not well described with the geophysically motivated functions just described. The uniform B-splines in term (7) are symmetric and have compact support. The set of B-splines decompose a signal into a series of recoverable, transient components of ground deformation (Figure 2). On the other hand, the time integral of B-splines, term (8), are one-sided, and decompose a signal into multiple non-recoverable, transient components, with each component akin to a slow step (Figure 2). The temporal frequencies of the transient deformation captured by each of the B-spline representations is given by the knot spacing, and as the knot spacing becomes smaller,

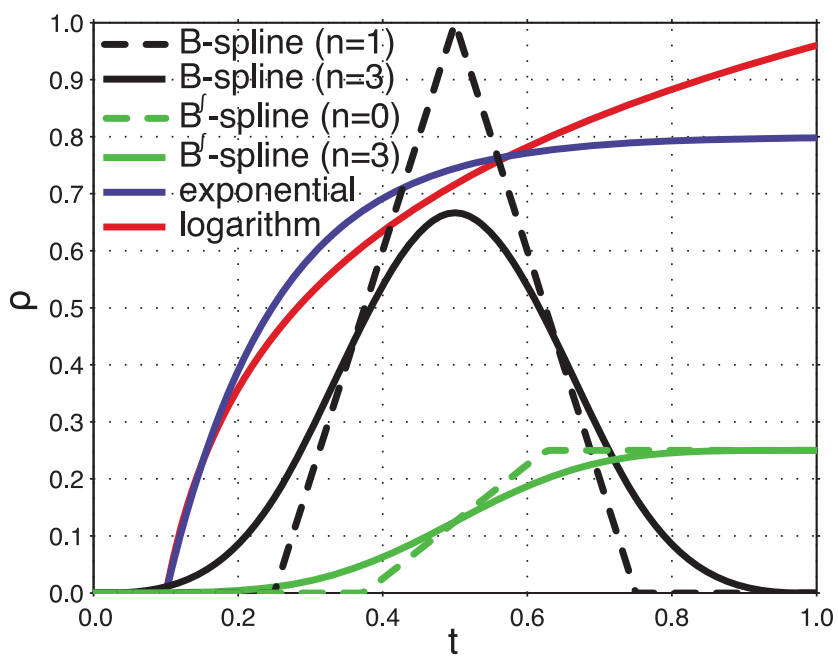

Figure 2. Basis functions used in the time-dependent parametrization of ground deformation. B-splines and $\mathrm{B}^{\int_{-}}$ splines have uniform knot spacing of 0.25 , and only the basis centered at 0.5 is shown. 
higher frequency deformation is captured. The non-uniform B-splines in term (9) are asymmetric, and can approximate one-sided deformation with the appropriate knot spacings. Non-uniform B-splines may be useful for describing transient deformation when one knows the approximate times of transient deformation, but the exact functional form is not known. Alternatively, non-uniform B-splines are useful if one wishes to tie the parametrization to the radar acquisition times. For example, the piecewise linear deformation model in the SBAS method [Berardino et al., 2002], can be replicated using zero-order non-uniform B-splines located at the acquisition times. For brevity, we refer to the integrals of $\mathrm{B}$-splines as $\mathrm{B}^{\int}$-splines.

[12] Each set of terms in (7)-(9) are a redundant and overcomplete set of basis functions. There may be a significant trade-off between generalized B-spline-based functions and the other functions (e.g., periodic terms). We stress that any particular inference of function coefficients is not unique when using a set of non-orthogonal, over-complete, and potentially redundant basis functions. However, if one is not necessarily interested in the values of the coefficients in terms (2)-(9) themselves, and more interested in the reconstruction of $F_{\rho}(\Omega, t)$, it is of minor consequence that the functions included in any realization of $F_{\rho}(\Omega, t)$ are not orthogonal. On the other hand, there may be some instances where the values of the coefficients are of interest, where one would most likely either remove that component of the deformation or use the inferred coefficients in a geophysical model. An example of the first case is using inferred amplitudes of sinusoidal terms to filter seasonal ground variations from the time-dependent deformation. Alternatively, in the second case, the amplitudes of steps might be used directly to constrain models of coseismic deformation. When using a non-orthogonal set of functions to represent the time-dependence of ground deformation, care should be taken to choose the regularization appropriate for the given non-orthogonal functional representation and the interferogram catalog. We purposely include a non-orthogonal set of functions in our representation of $F_{\rho}(\Omega, t)$, and allow a flexibility of regularization. Each geophysical target likely represents a unique case, and our formulation is open to testing multiple options.

\subsection{Interferometric Measurements}

[13] Equation (1) describes time-dependent ground position relative to some reference point. However, InSAR measures discrete differences in LOS deformation over given time spans. We define $\Delta \rho_{\alpha \beta}(\Omega)$ to be the difference in phase at a pixel located at $\Omega$ in an interferogram spanning times $t_{\alpha}$ to $t_{\beta}$. It is important that all of the interferograms are processed on a common geometry, and thus we reference all interferograms to the geometry of a common SAR image during processing. The InSAR measurements can be represented as

$$
\Delta \rho_{\alpha \beta}(\Omega)=\left[F_{\rho}\left(\Omega, t_{\beta}\right)-F_{\rho}\left(\Omega, t_{\alpha}\right)\right]+\mathcal{R}_{\alpha \beta}(\Omega)+\mathcal{N}_{\alpha \beta}(\Omega)
$$

where $\mathcal{R}_{\alpha \beta}(\Omega)$ describes long wavelength errors due to incorrect estimates of the interferometric baselines (i.e., orbital errors), and $\mathcal{N}_{\alpha \beta}(\Omega)$ describes additional noise contributions in the interferometric measurement.
[14] Orbital errors can be estimated either in the processing stage [e.g., Simons and Rosen, 2007], or by removing an estimate of $\mathcal{R}_{\alpha \beta}$ from the interferograms prior to determining the time-dependent deformation [e.g., Pritchard et al., 2006; Pritchard and Simons, 2006; Shirzaei and Walter, 2011]. Both methods implicitly assume that the orbital errors can be estimated independently from the ground deformation; however, this assumption is not always ideal, especially for longer wavelength deformation signals. It is also possible to use independent data, usually GPS observations, to either re-estimate the interferometric baseline or to constrain the longest wavelengths of the physical model [e.g., Peltzer et al., 2001; Simons et al., 2002; Lundgren et al., 2001], although we do not use such methods here.

[15] We approximate $\mathcal{R}_{\alpha \beta}$ as the spatial polynomial

$$
\mathcal{R}_{\alpha \beta}(\Omega)=a_{\alpha \beta}+b_{\alpha \beta} x+c_{\alpha \beta} y+d_{\alpha \beta} x y+\cdots
$$

In practice $\mathcal{R}_{\alpha \beta}$ is often taken to be a bi-linear function with four coefficients [e.g., Pritchard et al., 2006; Pritchard and Simons, 2006]. In some studies it may be appropriate to approximate $\mathcal{R}_{\alpha \beta}$ as a plane with three coefficients [e.g., Shirzaei and Walter, 2011] or a constant offset. We note that using equation (11) implicitly lumps together all longwavelength processes that could cause spurious phase ramps, not just orbital error. $\mathcal{N}_{\alpha \beta}(\omega)$ includes propagation delays due to atmospheric or ionospheric perturbations (i.e., "path delays" [Massonnet et al., 1993]), and noise due to temporal decorrelation of the reflective properties of the ground. Atmospheric path delays can be considered to be composed of a regional tropospheric stratification, plus a more spatially complex component which describes the impact of atmospheric and ionospheric dynamics [e.g., Delacourt et al., 1998; Cavalié et al., 2007]. The multiscale approach we outline here lends itself to a robust estimation of tropospheric path delays for a simple model in which the delays are correlated to topography [Lin et al., 2010]. However, exploring approaches to estimating the atmospheric contribution to the interferometric measurements along with the spatiotemporal deformation is left to a future study. For now we consider the path delays as some undefined data noise.

[16] Note that we do not need to include unknown offsets to tie together subsets of the stack that are not connected by interferograms (i.e., disconnected interferograms in perpendicular baseline and time space, referred to as $B_{\perp}$ space), as long as the temporal parametrization in $F_{\rho}$ does not depend on the acquisition times.

\subsection{Single Pixel Demo}

[17] Before discussing the spatial aspect of MInTS and the inversion procedure, we illustrate our temporal parametrization using a synthetic displacement time series at a single point (i.e., in a single pixel). We also use this synthetic example to highlight the differences between our timedependent parametrization and an assumption of constant deformation rate between SAR acquisition times. The synthetic deformation consists of a linear-rate, an instantaneous offset at time $t_{o}$, a logarithmic deformation following the offset, a non-instantaneous offset during the logarithmic 
a)

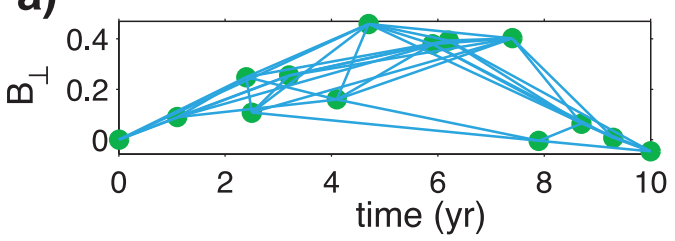

b)

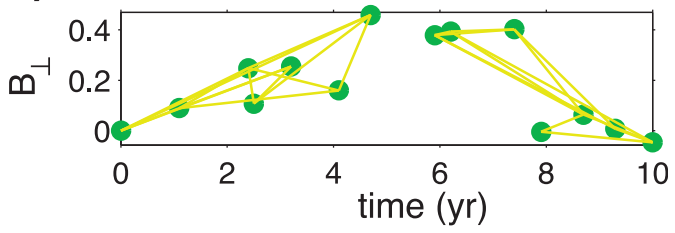

c)

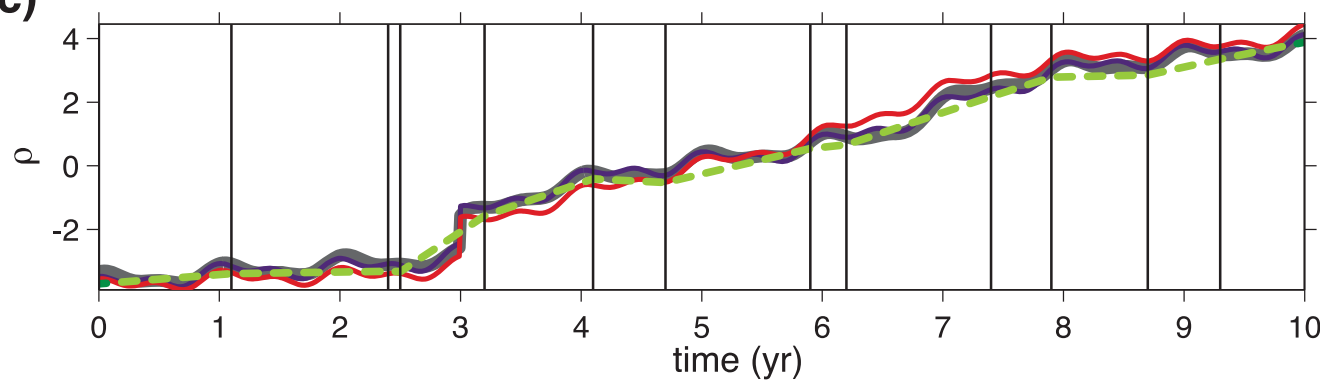

d)

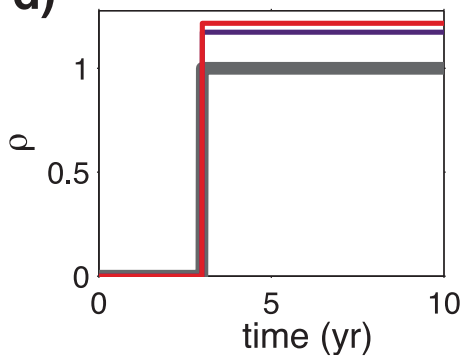

e)

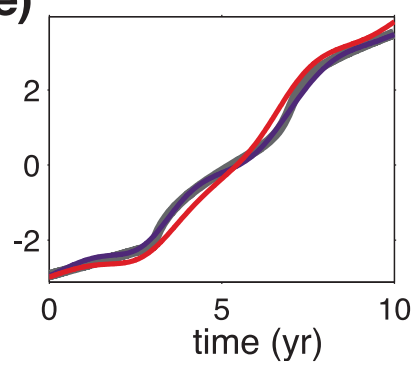

f)

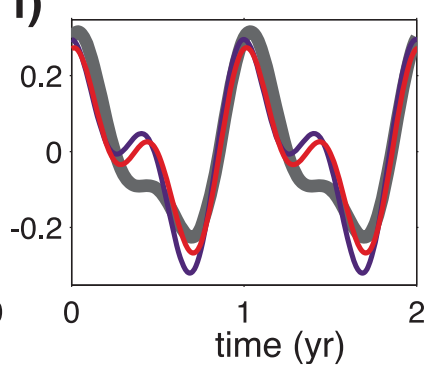

Figure 3. Two synthetic InSAR stacks: (a) one fully connected and (b) one disconnected. Green dots in Figures $3 \mathrm{a}$ and $3 \mathrm{~b}$ are synthetic SAR acquisition times, and $B_{\perp}$ values are for illustration purposes only. (c-f) Synthetic ground deformation (thick gray solid line) for a single position, and the recovered signals using the connected stack (blue line) or the disconnected stack (red line), assuming a time-parametrization as described in the main text. Also shown is the signal recovered from the connected stack assuming a linear deformation rate between each acquisition time (green dashed line; shown in Figure 3c only). Figure $3 \mathrm{c}$ is the total signal, Figure $3 \mathrm{~d}$ is the offset, Figure $3 \mathrm{e}$ is the linear rate, logarithmic decay and slow-step combined, and Figure $3 \mathrm{f}$ is the annual and semi-annual signals. Note that MInTS analysis is insensitive to absolute position, and the signals are arbitrarily shifted so that their means are zero.

decay, and annual and semi-annual seasonal components (Figure 3). We construct the synthetic $\Delta \rho$ samples, using two sample interferometric stacks based on a random temporal sampling (i.e., synthetic SAR acquisition times). In the first stack, we assume that a $\Delta \rho$ measurement is made in all pairs of acquisitions (Figure 3a), and in the second stack, we assume that there are no $\Delta \rho$ measurements spanning the center of the synthetic time series (Figure $3 b$ ). We refer to the first as the "connected stack", and the second as the "disconnected stack". We add $20 \%$ Gaussian noise to the synthetic $\Delta \rho$ measurements. We then invert the $\Delta \rho$ measurements in the two stacks for a time-parametrization composed of a constant offset at $t_{o}$, annual and semiannual seasonal components, and a collection of ten 3rd order $\mathrm{B}^{\int}$-splines. We damp the coefficients of the timedependent parametrization, and we use a ten-fold crossvalidation to determine the optimal penalty parameter (the inversion technique is fully described in section 2.7). For both the connected and disconnected stacks, we recover the total deformation signal well (Figure 3c). We also recover the magnitude of the offset at $t_{o}$ (Figure 3d), and the largest amplitude variation of the seasonal signals (Figure 3f) fairly well. The $\mathrm{B}^{\int}$-splines approximate the combined effect of the linear rate, the logarithmic decay, and the slow step (Figure 3e).

[18] For comparison, we also invert the connected stack for a constant rate of deformation between each of the synthetic SAR acquisition times. This piecewise linear time parametrization captures the overall trend of the synthetic signal, and resolves a change in rate coincident with the offset and start of the logarithmic decay (Figure 3b). However, using the piecewise linear time parametrization, it is difficult to resolve the structure of the deformation signal following the instantaneous offset. Without being able to identify the approximate contribution of various types of deformation (e.g., seasonal signals, offsets), it would make it difficult to clean those contributions from the total deformation.

[19] The recovery of a synthetic time-dependent signal depends on the particular choice of the synthetic SAR acquisition times; however, in numerous cases of random sampling of the synthetic time series, we found that we were able to recover the independent components of deformation 
fairly well. An exception is if the time-spans bracketing the instantaneous offset are long, then the inferred offset tends to be overestimated, and the rates immediately following the offset tend to be underestimated. Likewise, if the synthetic SAR acquisition times are at similar times in each synthetic year, the annual and semi-annual signals are not recovered well. It is important to note that these synthetic SAR acquisition times are fairly evenly distributed throughout the total time-span. In section 2.7, we discuss methods for additionally damping the time parametrization during times when there are sparse acquisitions.

\subsection{Data Covariances}

[20] The full data covariance is constructed of three primary parts: (1) the variance of the phase difference at a given pixel, (2) the intra-interferogram pixel-to-pixel covariance, primarily due to ionospheric and tropospheric path delays, and (3) the inter-interferogram covariances associated with use of a single common image in two separate interferometric pairs [Emardson et al., 2003]. As is commonly done, we assume that intra-interferogram pixel-to-pixel covariance depends only on the relative distance between two given pixels [Hanssen, 2001; Emardson et al., 2003; Lohman and Simons, 2005]. The inter-interferogram covariance can be derived analytically, while the intra-interferogram pixel covariance is found empirically [Emardson et al., 2003]. Following Emardson et al. [2003] and Lohman and Simons [2005], we define the covariance matrix between pixel $i$ in interferogram $(\alpha \beta)$ and pixel $j$ in interferogram $(\xi \zeta)$, as

$$
C_{i j}^{(\alpha \beta)(\xi \zeta)}=\frac{1}{2}\left[\sigma_{e}^{2}-\frac{1}{2}\left(1-\delta_{i j}\right) e^{L_{i j} / L_{c}}\right]\left(\delta_{\alpha \xi}+\delta_{\beta \zeta}-\delta_{\alpha \zeta}-\delta_{\beta \xi}\right),
$$

where $\sigma_{e}^{2}$ is the variance of a single pixel, $\delta_{i j}$ is the Kronecker delta, $L_{i j}$ is the distance between the pixels, and $L_{c}$ is a characteristic scale over which pixels are correlated in a single interferogram. For two pixels within a single interferogram, $\delta_{\alpha \zeta}=\delta_{\beta \xi}=0, \delta_{\alpha \xi}+\delta_{\beta \zeta}=2$, and the right-hand side of equation (12) becomes either $\sigma_{e}^{2}$ (variance of a single pixel) or $\sigma_{e}^{2}-\frac{1}{2} e^{L_{i j} / L_{c}}$ (covariance between two pixels). For interferograms with one common scene, only one $\delta_{i j}$ is nonzero, and the covariance is $\pm 1 / 2$ the covariance in a single interferogram [Emardson et al., 2003]. For interferograms with no common scenes, the covariance is zero.

[21] In the case of redundant interferograms in $\mathrm{B}_{\perp}$ space, for instance with three interferograms formed from any three acquisitions, the data covariance matrix is singular. The singularity arises from the fact that the temporal portions of the covariance matrix are positive semi-definite. This semidefiniteness can be understood since the dual of the connectivity matrix of a closed graph is not uniquely defined [Whitney, 1932], and the temporal covariance of interferometric measurements is simply $F C_{\mathrm{SAR}} F^{T}$, where $C_{\mathrm{SAR}}$ is a diagonal covariance matrix of a single radar acquisition and $F$ is the connectivity matrix of the interferometric stack. (Mapping the SAR covariance matrix with the connectivity matrix results in the identical temporal covariances of interferograms as derived by Emardson et al. [2003].) To account for the positive semi-definiteness of the temporal portions of the covariance matrix, we use the SVD pseudoinverse of the covariance matrix in the least squares inversion [e.g., Krzanowski et al., 1995; Bondár and McLaughlin, 2009].

[22] In the case that the spatial correlation length-scale is shorter than the interferogram pixel dimensions, one could treat each pixel independently. In this case, the data covariance matrix will then only contain information regarding the correlations due to interferograms sharing common scenes [e.g., Emardson et al., 2003], and considering each pixel independently would be appropriate. At the other extreme, where the spatial correlation length-scale is comparable to the interferogram dimension, the entire data covariance matrix should be considered in the inversion for the spatiotemporal deformation. For $M$ interferograms, each with $N$ pixels, $C_{i j}^{(\alpha \beta)(\xi \zeta)}$ is an $N M \times N M$ matrix (assuming no regions of low interferometric correlation). For $N=\mathcal{O}\left(10^{4}\right)$ pixels and $M=\mathcal{O}(10)$ interferograms, $C_{i j}^{(\alpha \beta)(\xi \zeta)}$ would have $\mathcal{O}\left(10^{10}\right)$ elements, although in general, $C_{i j}^{(\alpha \beta)(\xi \zeta)}$ may be much larger. A matrix of this size would be computationally expensive to invert, if possible at all; however, the sparseness of the data covariance could be utilized in the inversion scheme.

[23] Lohman and Simons [2005] found that the correlation length scale in interferograms was on order of tens of kilometers, so in most cases smaller than the interferogram size, but much larger than the pixel size. Lohman and Simons [2005] also found that $L_{c}$ inferred from interferometric data not surprisingly decreased when the best fit spatial bi-linear ramp was removed from the interferogram. Likewise, the extent of spatial correlation is decreased when the interferograms are successively high-pass filtered. Motivated by this observation, we base MInTS on a spatial filter bank constructed using 2D Meyer wavelets.

\subsection{Multiscale Spatial Decomposition}

[24] We represent the 2D spatial discrete wavelet decomposition of a given unwrapped interferogram as

$$
\Delta \rho_{\alpha \beta}(\Omega)=\sum_{a, b} r_{a, b}^{\alpha \beta} \psi_{a, b}(\Omega)
$$

where $\psi_{a, b}(\Omega)$ are 2D spatial wavelets of scale $a$ and position $b$, and $r_{a, b}^{\alpha \beta}$ are the corresponding wavelet coefficients for the interferogram spanning $t_{\alpha}-t_{\beta}$. We similarly represent the spatial discrete wavelet transform of $F_{\rho}(\Omega, t)$ as

$$
F(\Omega, t)=\sum_{a, b} \mathcal{F}_{a, b}(t) \psi_{a, b}(\Omega),
$$

where $\mathcal{F}_{a, b}(t)$ represents the time-dependent wavelet coefficients in the parametrization of the time-dependent deformation. For example, if the time-dependent deformation is parameterized by a linear-rate only, $\mathcal{F}_{a, b}(t)$ represents the coefficients of the spatial wavelet decomposition of the velocity field. If the time-dependent deformation is parameterized by a collection of uniform B-splines, then

$$
\mathcal{F}_{a, b}(t)=\sum_{i \in \mathcal{I}_{U B}} \tilde{\kappa}_{a, b}^{i} B_{n}\left(t-t^{b}\right),
$$


a)

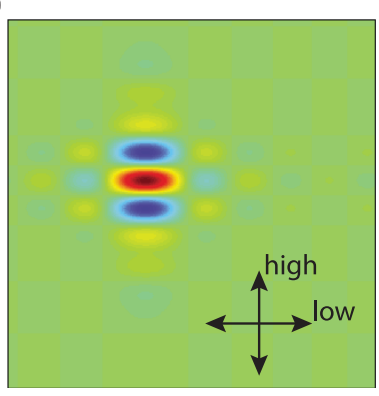

b)

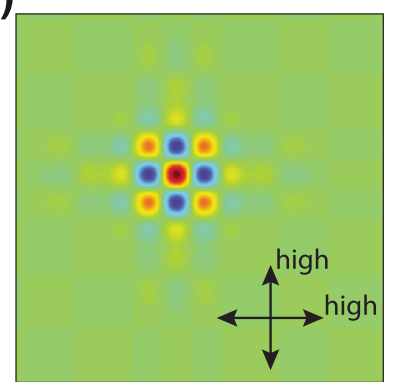

c)

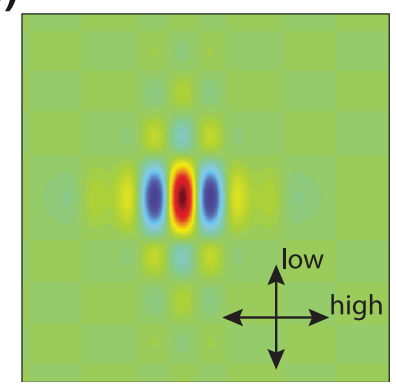

Figure 4. Meyer wavelets in the spatial domain, representing the (a) high-low, (b) high-high, and (c) low-high frequency bands in the two spatial directions.

where $\tilde{\kappa}_{a, b}^{i}$ are the coefficients of the discrete wavelet transform of $\kappa_{i}$ :

$$
\kappa_{i}(\Omega)=\sum_{a, b} \tilde{\kappa}_{a, b}^{i} \psi_{a, b}(\Omega)
$$

In the present implementation of MInTS, we use 2D Meyer wavelets [Mallat, 2008]. In the 2D wavelet transform, there are three components at each scale (Figure 4), representing the high-low, low-high, and high-high frequency bands in the 2D spatial frequency domain [e.g., Mallat, 2008].

[25] For a distance-dependent covariance model, Meyer wavelets have the benefit that the covariances between wavelet coefficients in a single interferogram are negligible (Appendix A). Essentially the discrete wavelet transform decorrelates the spatial part of the data covariance matrix. The decorrelating power of the wavelet transform overcomes the computational challenge associated with using the full data covariance matrix, and thus we can determine the timedependence of each wavelet coefficient independently. In essence, because the wavelets are supported over a finite number of pixels, the wavelet coefficients themselves inherently include information about the underlying spatial data covariances at any given spatial scale. While we use Meyer wavelets in this paper, the reduction of the covariances between coefficients is a general benefit of wavelet decomposition, although the covariances between wavelet coefficients may be larger with other wavelet transforms. In some situations, it might be desirable to use other wavelets, for instance to account for anisotropic spatial correlations [e.g., Knospe and Jòsson, 2010], or to use phase-preserving wavelets. The latter is important if unwrapping is to be attempted simultaneously with the time-dependence estimation. The wavelet approach also provides a consistent method for space-time interpolation of holes (i.e., regions of low interferometric correlation) in individual interferograms.

\subsection{Interferogram Wavelet Transformation}

[26] A general requirement of discrete wavelet transforms (DWT) using dyadic wavelets is that the dimension of the image must be base two. For Meyer wavelets the size must be $2^{J+3} \times 2^{J+3}$ pixels, where $J$ is the maximum scale of the wavelet transform. Wavelet scale 1 refers to the highest spatial frequency, and scale $J$ is the lowest. After removal of scales $1-J$, there is a remaining image representing the information at the lowest spatial frequencies. There are three components at each wavelet scale $1-J$, with only one component for the low-pass residual [e.g., Mallat, 2008]. As interferograms are not constrained to be of base-two size when processing, we expand each interferogram to a basetwo dimension by mirroring, which also avoids possible edge-effects in the transformation (Figures 5a and 5b). Additionally, the DWT requires that the interferograms do not contain any holes. We interpolate within masked regions associated with low interferometric correlation in the unwrapped interferograms using an inpainting algorithm developed by John D'Errico and freely available as the Matlab function inpaint nans.m from the Matlab Central file exchange (Figure 5c). To construct the wavelet coefficients $r_{a, b}^{\alpha \beta}$ of each interferogram, we use the Meyer DWT included in the WaveLab package implemented in Matlab [Buckheit and Donoho, 1995] (Figure 5d). As we do not want the interpolated values within the interferogram holes to affect the resulting model of time-dependent deformation, we calculate weights for every wavelet coefficient based on the degree to which the associated wavelet is supported by interpolated values (Figure 5e). For instance, in the event that a wavelet at a given scale and location in an interferogram does not overlap any holes, the weight would be one. On the other hand, if wavelet is supported entirely by interpolated values, the weight would be zero (Figure 5e). We then use these weights in the inversion for timedependent deformation as described below.

\subsection{Inversion Method}

[27] We estimate the temporal behavior of a given wavelet by solving the least squares solution that minimizes

$$
\phi=\left\|G m_{a, b}-y_{a, b}\right\|_{2}^{2}+\lambda^{2}\left\|H m_{a, b}\right\|_{2}^{2},
$$

where $\|\cdot\|_{2}$ is the L2-norm, the vector $y_{a, b}$ represents the wavelet coefficients at a given scale $a$ and location $b$ in all of the interferograms, and the vector $m_{a, b}$ represents the wavelet coefficients of the parameters of the time-dependent deformation in $\mathcal{F}_{a, b}(t) . G$ is the design matrix based on the choice of $F(\Omega, t), H$ is a model regularization matrix, and $\lambda$ is the regularization penalty parameter. We find the weighted least squares solution as

$$
\widetilde{m}_{a, b}=\left(G^{T} W^{T} C_{t}^{\dagger} W G+\lambda^{2} H\right)^{T} G^{T} W^{T} W y_{a, b},
$$



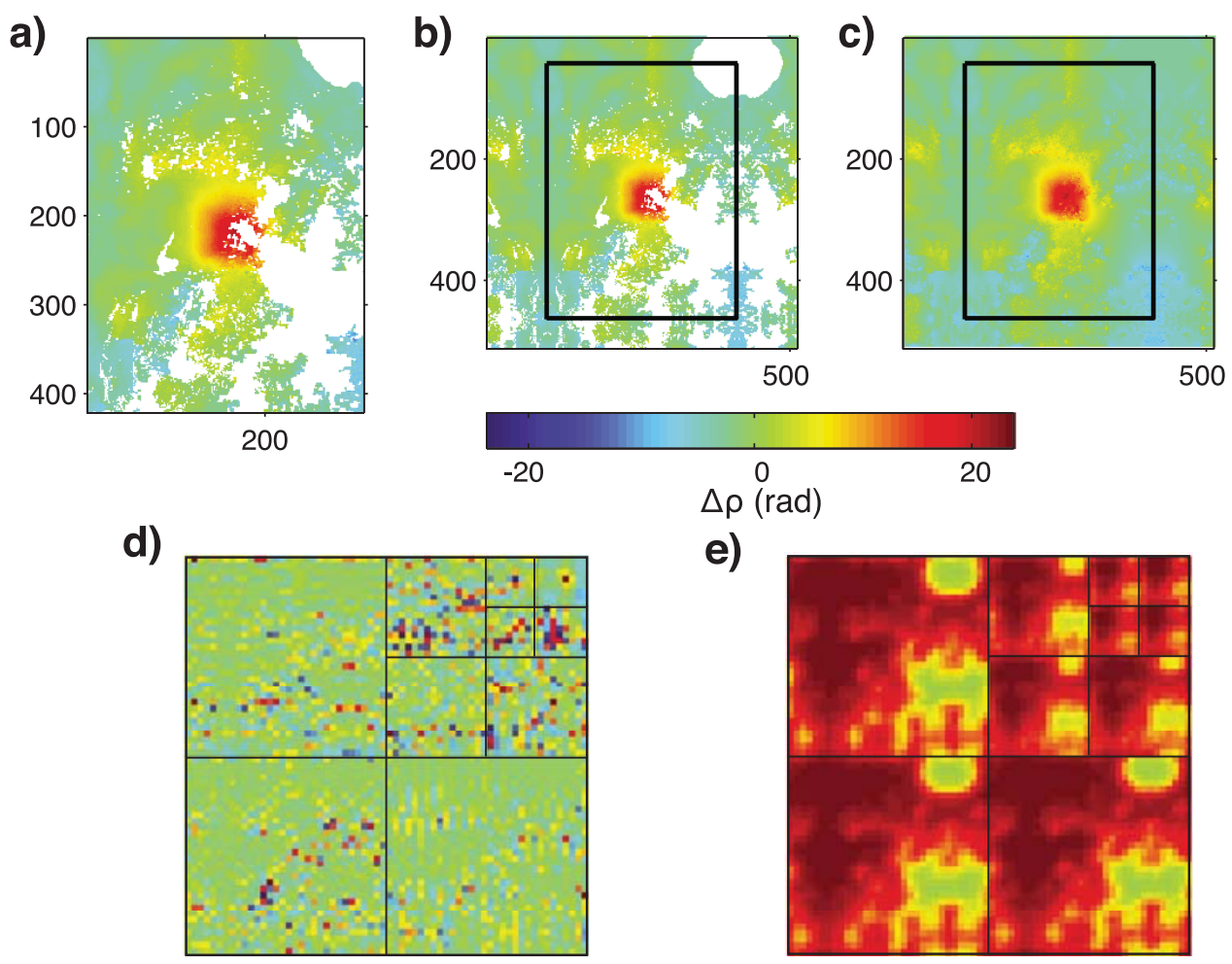

Figure 5. (a) Unwrapped interferogram with regions of low interferometric correlations masked out. (b) Interferogram mirrored to expand to a base-two size; solid line is the outline of the original interferogram in Figure 5a. (c) Interferogram with the masked regions filled using an inpainting algorithm. (d) The wavelet coefficients for the image in Figure 5c, only scales greater than 4 and the remaining low-pass image are shown. (e) The calculated weights on the wavelet coefficients in Figure 5c, signifying the relative amount of support of each wavelet over decorrelated regions; color scale is from green to red, signifying wavelets with zero weight to a weight of one.

where $C_{t}^{\dagger}$ is the pseudo-inverse of the temporal component of the data covariance matrix, and $W$ is the weighting matrix defined in the section 2.6. We solve equation (18) separately at each scale and location, and we retain only the temporal components of the data covariance matrix as the covariances between wavelet coefficients at neighboring locations and scales are negligible (see Appendix A). We use the SVD pseudo-inverse as the covariance matrix is positive semidefinite and singular whenever there are redundant interferogram pairs [e.g., Krzanowski et al., 1995; Bondár and McLaughlin, 2009]. The diagonal weighting matrix, $W$, reflects the degree to which a given wavelet is supported by measured as opposed to interpolated phase differences. It is important to note that $W$ is distinct for each scale and location, although for brevity we do not write $W$ as $W_{a, b}$.

[28] The type of regularization in $H$ should be chosen based on the properties of the time-dependent basis functions. For instance, when using B-splines, it might make most sense to choose to enforce a smoothness constraint, either on the model parameters or on the time-dependent deformation. On the other hand, when using $\mathrm{B}^{\int}$-splines, it might make most sense to damp the $\mathrm{B}^{\int}$-spline amplitudes, so that any given $\mathrm{B}^{\int}$-spline will only have a non-negligible amplitude if a transient offset is required by the data in the associated time period. In our toolbox, the choice of regularization is left to the user. We solve equation (18) using the
Tikhonov regularization implemented in the Regularization Tools Matlab toolbox [Hansen, 1994, 2007]. To determine $F(\Omega, t)$, we take the inverse discrete wavelet transform (IDWT) of each of the model parameters in $\widetilde{m}_{a, b}$. The IDWT of the model wavelet coefficients results in the spatial fields of the amplitudes in the time-dependent function $F(\Omega, t)$.

\subsubsection{Cross-Validation}

[29] The penalty parameter $\lambda$ is chosen using $n$-fold crossvalidation [e.g., Stone, 1974; Geisser, 1975]. In crossvalidation, the data are randomly split into two sets, a learning set and a testing set. Only the data in the learning set are inverted for various choices of $\lambda$, with the estimated model found for each choice of $\lambda$ tested against the remaining data in the testing set. The optimal $\lambda$ is such that the model estimated from the learning set produces the best fit to the data in the testing set. It is important that the learning and testing sets are formed such that every datum is in either the learning or testing sets, but not both, that the data in the testing set represents a non-biased test of the predictability of the estimated model, and that the data in the learning and testing sets are independent [Picard and Cook, 1984]. In $n$-fold cross-validation, the data is partitioned into $n$ nearly equal size learning sets, each with an associated testing set. The optimal penalty parameter is then taken as the $\lambda$ in which the $n$ estimated models best fit the data in the associated testing sets. If $n$ is equal to the 
number of data, $n$-fold cross-validation is just leave-one-out cross-validation.

[30] We form the cross-validation sets based on the radar acquisitions, not the interferograms. We randomly select radar acquisitions, and all interferograms formed from those acquisitions forms a learning set. The associated testing set is composed of all interferograms not in the learning set. For example, in 10-fold cross-validation the radar acquisitions are split into ten nearly equal subsets. All of the interferograms formed by the acquisitions in each subset then forms ten learning sets, with the remaining interferograms forming ten testing sets. Forming the learning/testing sets based on the acquisitions minimizes, but does not entirely avoid, the dependence between the learning and testing sets in most cases. In our experience, for $N$ acquisitions, $n$-fold cross-validation is most stable when $n / N \approx 1 / 3$.

[31] In an interferometric stack, there can be several million sets of wavelet coefficients at every scale and location. Cross-validating for the optimal penalty parameter independently at each scale and location can be computationally demanding. Moreover, one may expect that the amount of regularization required at nearby location might be similar. The computational time of the cross-validation may be decreased by only cross-validating at a sub-sample of locations at each scale, and then inverting at all scales and locations assuming that the optimal penalty parameter in a given scale varies smoothly in space. Likewise, the crossvalidation for optimal $\lambda$ can be skipped at locations and scales where wavelets are mostly supported by interpolations within decorrelated regions in all interferograms.

\subsubsection{Variable Shape Smoothing}

[32] In some parameterizations of time-dependent deformation constrained by a given InSAR stack, it is advantageous to apply differing degrees of regularization to different model parameters. For example, in the case when one parameterizes time-dependence with evenly spaced Bsplines, an uneven distribution of SAR acquisitions may result in time spans that are less resolved. One can always tie the time parametrization to the acquisition dates; however, using constant time sampling allows MInTS to use the same time parametrization for all regions, regardless of whether a given region may have low interferometric correlation in any given subset of interferograms. Using uniform time sampling with variable acquisition times, one should apply a large regularization to the model parameters that control components of deformation in periods of sparse or no SAR acquisitions.

[33] In order to regularize relatively unconstrained model parameters to a larger degree than more constrained parameters, we augment the model regularization by a diagonal variable shape smoothing matrix, $S_{p}$, which is based on an initial estimate of the model resolution matrix [Lohman, 2004]. The diagonals of the model-resolution reflect the relative resolution of each of the model parameters [e.g., Aster et al., 2005]. We estimate the model resolution using an SVD of the non-regularized inverse problem [Golub and van Loan, 1980], where we truncate the pseudo-inverse at the $p$ 'th singular value. We use this truncated pseudo-inverse to compute the model resolution matrix, $R_{p}$, and in index notation the diagonals of $S_{p}$ are

$$
S_{p_{i i}}=\left|1-R_{p_{i i}}\right|^{a} .
$$

The power $a$ determines the dynamic range of the variable shape smoothing, and we commonly take $a=1 / 2$. The degree to which less constrained model parameters are more heavily regularized compared to more constrained parameters is mainly controlled by $p$. In general, a smaller $p$ results in models that are rougher in times of sparse SAR acquisitions and a larger $p$ results in models that are rougher during times of dense acquisitions.

[34] When augmented by a model-resolution-based regularization, the total regularization in equation (18) is $H^{\prime}=S_{p} H$, where $H$ is the typical model regularization matrix. As $S_{p}$ is diagonal and $0<S_{p_{i i}}<1$, the regularization on each model parameter is equivalent to $\lambda_{i}^{2} H$, where $\lambda_{i}^{2}=\lambda^{2} S_{p_{i}}$ are composite penalty parameters specific to each model parameter. When the $i$ 'th model parameter is perfectly resolved, $\lambda_{i}=0$, and that model parameter is not subject to regularization. Alternatively, when the $i$ 'th model parameter is completely unresolved, $\lambda_{i}=\lambda$.

\section{Application to Long Valley, CA}

[35] To illustrate the application of MInTS, we consider a stack of interferograms of the Long Valley, CA, volcanic region. Several uplift events in Long Valley have been observed using ground based geodesy over the last few decades [Langbein et al., 1993, 1995; Langbein, 2003; Feng and Newman, 2009]. The largest was in late 1997, where 2-color electronic distance measurements (EDM) indicated short-lived rapid uplift of the resurgent dome in the Long Valley caldera [Langbein, 2003].

[36] We construct an interferometric stack using 24 ERS-2 and 15 Envisat SAR descending acquisitions, with a total of 92 interferograms (63 ERS-2 and 29 Envisat interferograms) spanning just over 16 years (Figure 6a). The interferograms are looked down eight times, equivalent to a resolution of about 196 and 160 meters in the range and azimuth directions, respectively. We unwrap the interferograms using the SNAPHU algorithm [Chen and Zebker, 2001]. The SNAPHU algorithm underestimates the regions of low interferometric correlation, for instance almost always unwrapping over Mono lake. Therefore, we calculate a common mask based on regions below a correlation coefficient of 0.25 in an interferogram formed from a one-day ERS pair (01-02 June 1996). We apply this common mask to all interferograms, in addition to those determined for each interferogram during unwrapping. We remove the best fit bi-linear ramp from each interferogram to account for long-wavelength signal we attribute to orbital errors.

[37] We follow the procedure outlined in section 2.6 to take the DWT of the interferogram stack. The time it takes to compute the DWT and calculate the weights of the wavelet coefficients of one interferogram depends on the interferogram size and the amount of interferometric decorrelation. For each of these interferograms, it takes a few minutes on a desktop computer with dual $3.2 \mathrm{GHz}$ quad-core CPUs. Each interferogram is independently processed, and thus the process is inherently parallelizable. For each stack of interferograms, the DWT and the weights only need to be computed once.

[38] We invert the stack assuming a time-dependent deformation model composed of 42 evenly spaced $\mathrm{B}^{{ } \text {- }}$ splines, and we regularize by damping the magnitude of the amplitudes of the $\mathrm{B}^{\int}$-splines. Since the SAR acquisitions are 

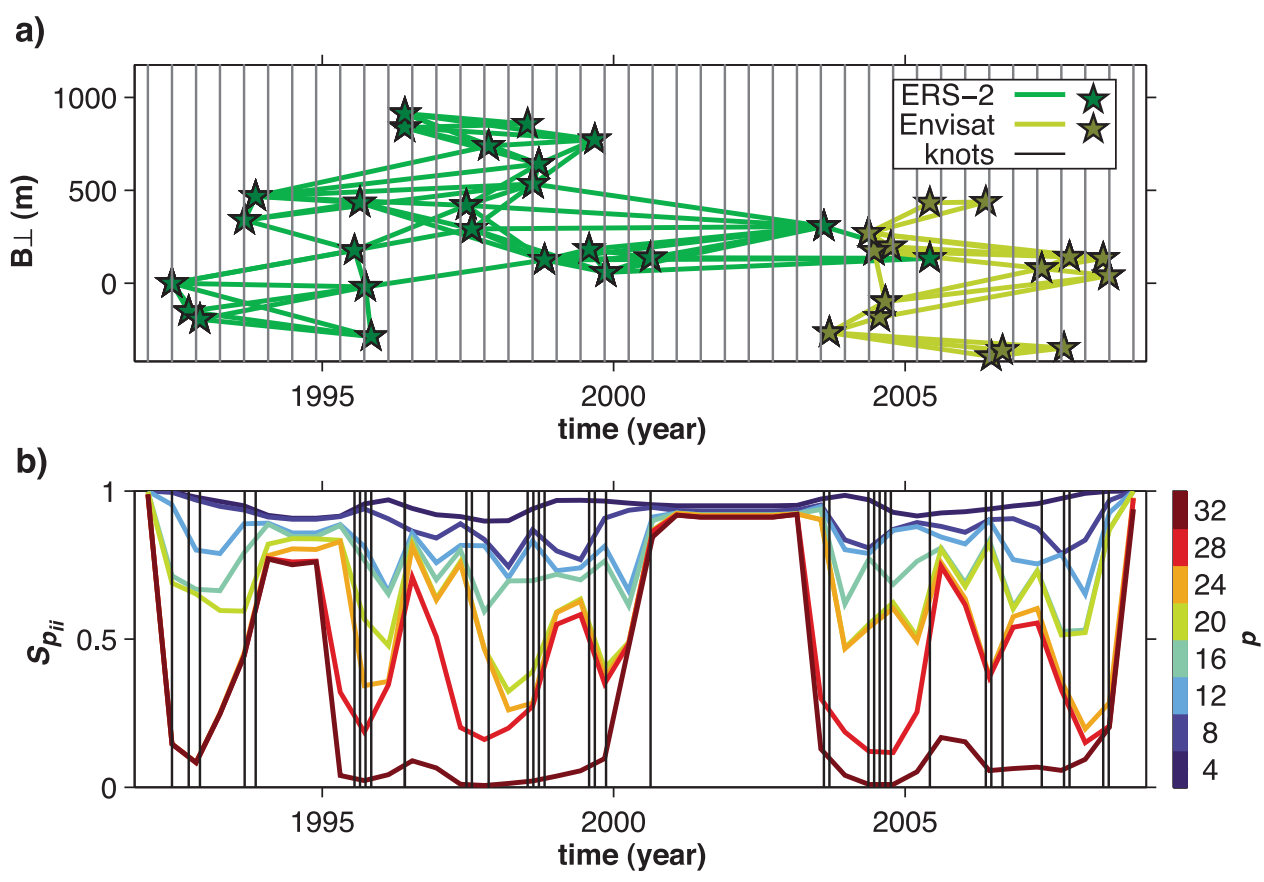

Figure 6. (a) Perpendicular baseline, $B_{\perp}$, plot for the Long Valley interferometric stack; stars indicate $B_{\perp}$ of each SAR orbit relative to the first SAR pass, and solid lines indicate interferograms. Gray vertical lines indicate the knot locations of the $\mathrm{B}^{\int}$-splines in the temporal parametrization of deformation in the Long Valley region. (b) Diagonals of the shape smoothing matrix, $S_{p_{i i}}$, for various truncations of the SVD pseudo-inverse at the $p$ th singular value. Black thin lines indicate the SAR acquisition times.

not evenly spaced in time (Figure 6a), we apply a variable shape smoothing in order to more heavily damp the $\mathrm{B}^{f_{-}}$ spline amplitudes during periods of no SAR acquisitions, relative to the amplitudes during periods of dense acquisitions. Three parameters control the model regularization in the inversion: (1) the penalty parameter, $\lambda,(2)$ the singular value truncation, $p$, of the pseudo-inverse used to determine $S_{p}$, and (3) the exponent in the variable smoothing, $a$. We use 12-fold cross-validation in order to determine the optimal $\lambda$ at each scale and location separately. We assume a common $p$ and $a$ in all inversions, since cross-validation on these two parameters is not well defined as the crosscorrelation objective functions do not have a clear minimum in terms of $p$ or $a$. The choice of $a$ has the smallest affect on the inversion results, and we use $a=1 / 2$. Recall that each model parameter is regularized by a composite penalty parameter, $\lambda_{i}^{2}=\lambda^{2} S_{p_{i i}}$ and using a small $p$ results in all of the model parameters being regularized almost uniformly (Figure 6b). As $p$ approaches the number of model parameters, $S_{p_{i i}}$ corresponding to model parameters during times that are densely sampled decreases (Figure 6b), and the model is largely non-regularized during those periods. We use $p=20$, although the results we present here are consistent with those using other values of $p$. A more formal approach to selecting the total regularization is left to a future analysis.

[39] MInTS analysis of this example stack takes just under 12 hours on the aforementioned computer, which includes cross-validated inversion of 262,088 sets of wavelet coefficients. In Figure 7, we show the cumulative LOS ground displacement determined in the MInTS analysis over four time periods, roughly corresponding to prior to the 1997 1998 uplift event, during the event, during the period of sparse radar acquisitions (cf. Figure 6a), and during the last four years of the stack time span. The spatiotemporal deformation is presented in the radar range and azimuth coordinates, and for these descending orbits, increasing range and azimuth is roughly westward and southward, respectively. The deformation is along the satellite LOS direction. We use a convention wherein increasing $\rho$ corresponds to ground deformation toward the satellite, which we loosely refer to as uplift. In Figure 8a, we show the timedependent deformation in three regions, roughly corresponding to the region of maximum uplift during the 1997 1998 uplift event (labeled A in Figures 7 and 8), the Casa Diablo geothermal field (labeled B), and north of Mammoth mountain (labeled C).

[40] The results show a steady uplift of the resurgent dome during 1993 to about mid-1997, followed by an abrupt increase in uplift rate until about mid-1998. The maximum uplift is to the eastern edge of the resurgent dome, and the location of maximum uplift shifts slightly to the south when the uplift accelerated during 1997-1998 (Figure 7). Following the uplift event, there is a slight subsidence of the Long Valley caldera to the west of the resurgent dome. We do not resolve the uplift event that occurred between 2002 and 2003, which was of smaller magnitude than the 19971998 event [e.g., Feng and Newman, 2009]. That we do not capture the smaller uplift is not surprising, as there are no SAR acquisitions during 2001 to 2004, and the model parameters are highly damped during this time period due 

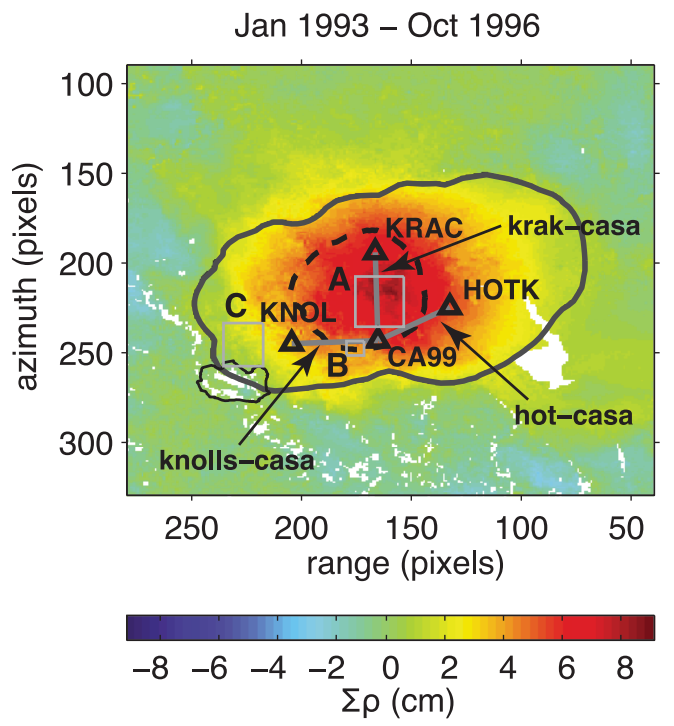

Jan 1999 - Oct 2003

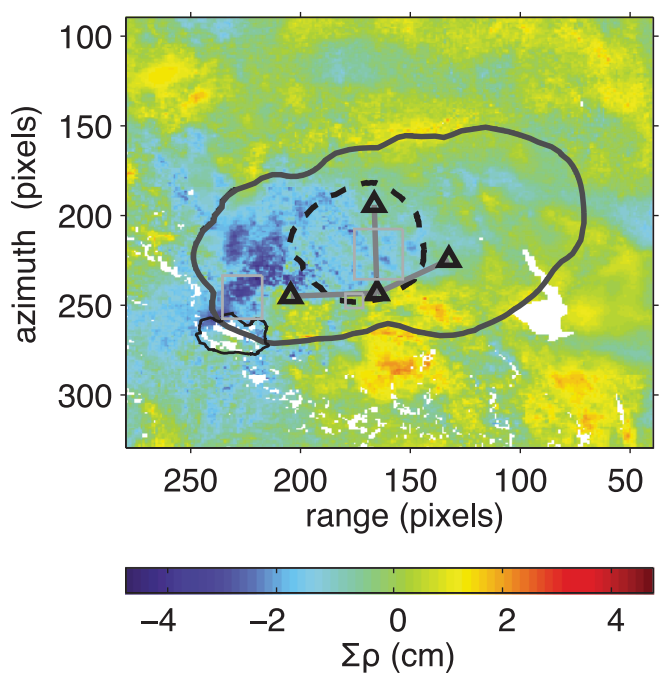

Jan 1997 - Oct 1998
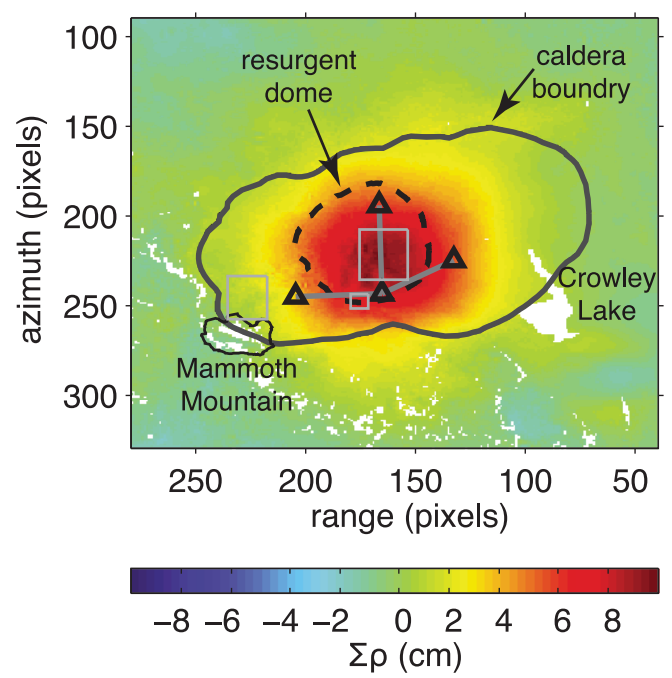

Jan $2004-$ Jul 2008

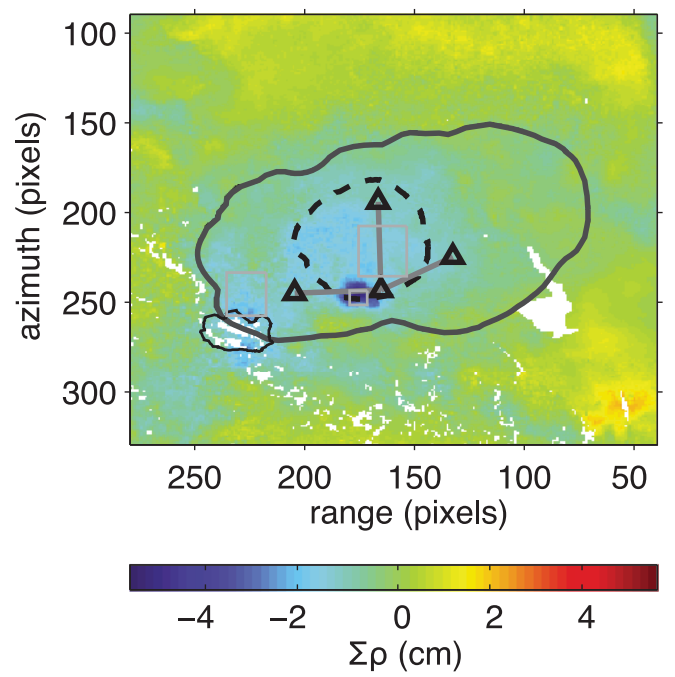

Figure 7. Cumulative LOS displacement, $\Sigma \rho$, over the indicated time periods, see main text for details of the parameters used in the MInTS analysis. Triangles show the locations of three continuous GPS stations, grey lines are select EDM baselines, and boxes labeled A, B, and C are where the time-dependent displacements are shown in Figure 8. Also indicated is Crowley Lake and the outlines of the Long Valley caldera (solid black line), resurgent dome (dashed black line), and Mammoth mountain (thick solid black line; geographic features are labeled in upper-right panel).

to the model-resolution-based variable shape smoothing (Figure 6). There is a slight subsidence over the resurgent dome during 2004-2008, and greater subsidence $(\approx 1 \mathrm{~cm} / \mathrm{yr})$ of the Casa Diablo geothermal plant.

[41] The results we obtain here are broadly consistent with those obtained using SBAS [Tizzani et al., 2007, 2009], although our results have higher spatial density. One notable difference is that we do not resolve the subsidence at the Casa Diablo geothermal field during the 1990's [Howle et al., 2003; Tizzani et al., 2007]. We do resolve that the Casa Diablo region is not uplifting as rapidly as in the rest of the caldera, but we do not resolve the subsidence over several kilometers that has been documented by leveling measurements [Howle et al., 2003]. The early subsidence in the Casa Diablo region is not apparent in the interferograms we consider here (see auxiliary material), ${ }^{1}$ which indicates that the Casa Diablo deformation was essentially smoothed over in the InSAR processing.

[42] The inferred spatiotemporal deformation is broadly consistent with the EDM observations (Figure 7b). EDM measures changes in distance between two points on the ground, whereas the recovered deformation from the InSAR

${ }^{1}$ Auxiliary materials are available in the HTML. doi:10.1029/ 2011JB008731. 
a)

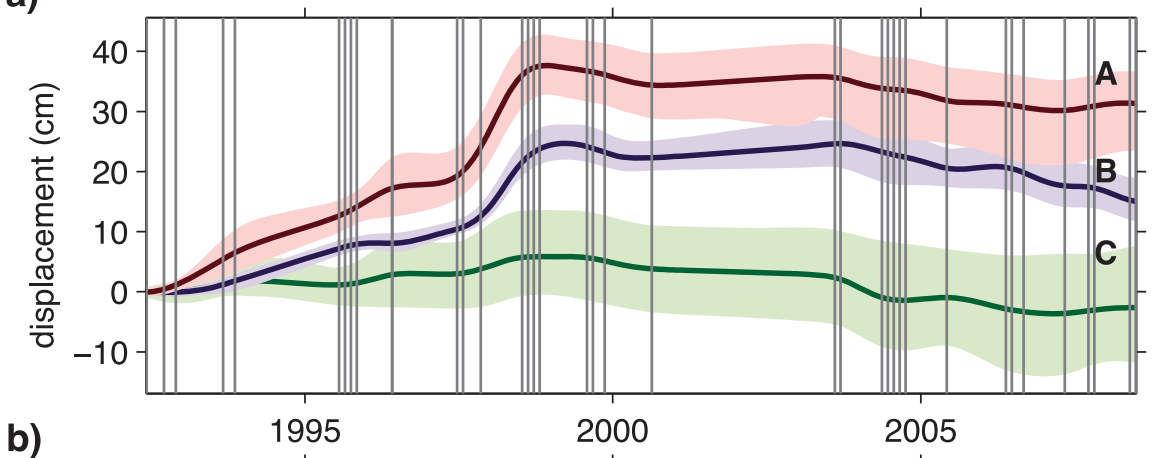

b)

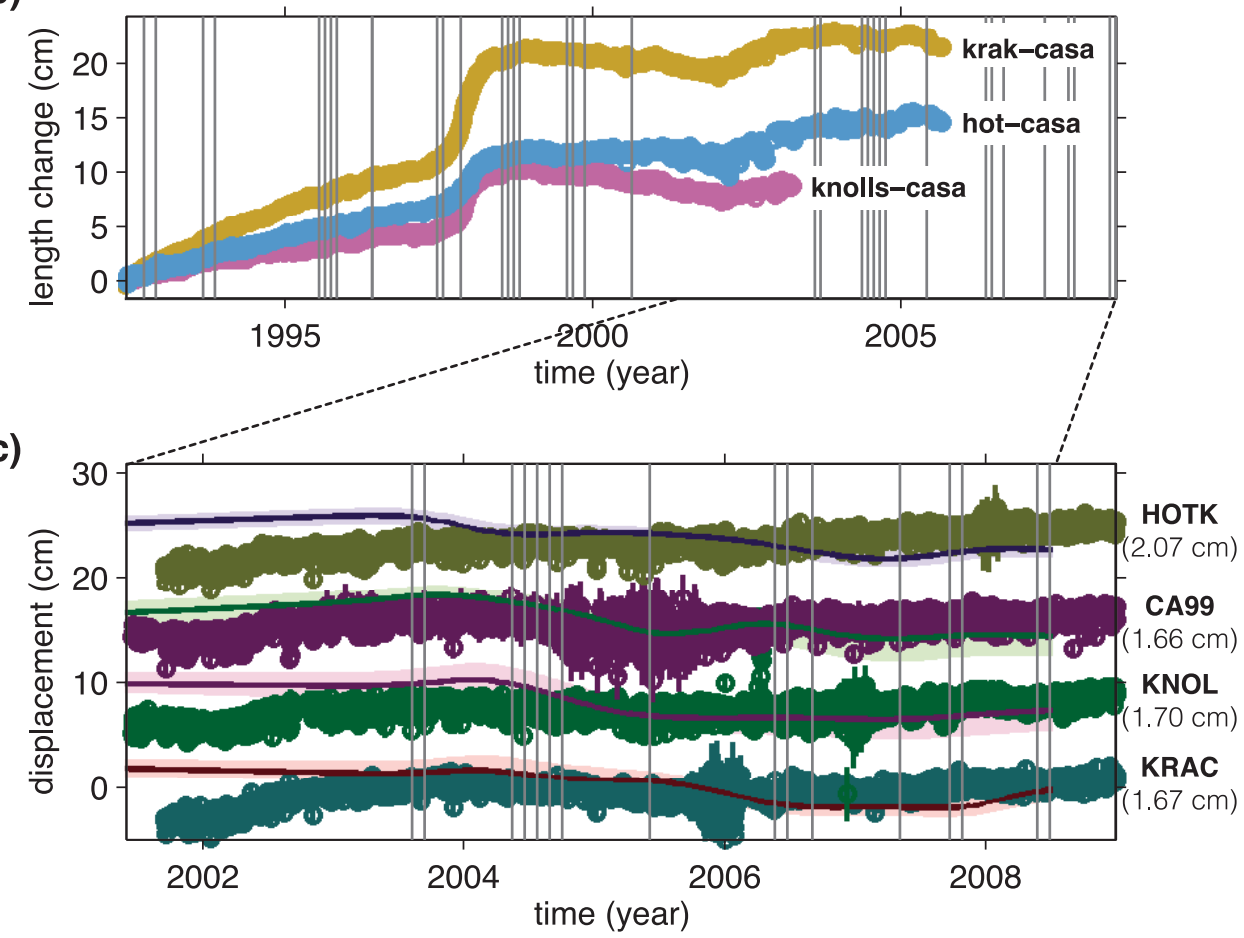

Figure 8. (a) Time series of LOS displacements determined from the Long Valley stack within the boxes $\mathrm{A}, \mathrm{B}$, and $\mathrm{C}$ shown in Figure 7; solid colored lines are the mean displacement and shaded regions indicate the total spread of displacements in each box. (b) Line length changes measured by two-color EDM along three baselines. (c) Displacements at four continuous GPS stations projected in the LOS. Solid lines and shaded region shows the average and variation in LOS displacements determined by MInTS analysis, over a $3 \times 3$ pixel region centered at each GPS station (the root mean square difference between the GPS and model is indicated). Vertical gray lines in all three panels are the SAR acquisition times, and locations of EDM baselines and GPS stations are shown in Figure 7.

stack is of ground deformation along the satellite LOS. Due to the differing nature of these two data sets, we do not attempt a direct comparison between our results and the EDM measurements. Continuous GPS measures the ground deformation in three components through time, and we show the projection of these measurements onto the LOS at four continuous GPS sites in Figure 8c. The continuous GPS records start in late 2001, and resolve a relatively small magnitude uplift from 2002 to 2004, followed by relatively steady deformation [Feng and Newman, 2009]. The along LOS deformation we constrain from the MInTS analysis is noisier than the GPS time series (Figure 8c).

[43] In the present version of MInTS, we do not use either the EDM or GPS data to constrain the spatiotemporal deformation. The spatiotemporal deformation is determined such that the predicted interferograms best fit the observed interferograms in the InSAR stack. There are systematic residuals that are correlated with the resurgent dome and caldera boundary (see auxiliary material). There is a residual signal in the center of the resurgent dome in several of the interferograms that is similar to the observed phase differences, and probably indicates un-modeled ground deformation. That there is a slight amount of un-modeled ground deformation apparent in the residuals indicates this spatiotemporal deformation field estimated from MInTS analysis is slightly too smooth in time. On the other hand, some of the residuals appear to be correlated with topography, and may indicate topographically correlated path delays due to 
tropospheric effects [e.g., Delacourt et al., 1998; Cavalié et al., 2007; Lin et al., 2010]. The topographically correlated residuals are most apparent in the Envisat interferograms, which capture a time during which there was less ground deformation than in the proceeding ten years.

\section{Future Directions}

[44] MInTS is a new tool for exploring crustal deformation using temporally deep collections of InSAR observations. The framework of MInTS also lends itself to being extended, for instance to include better treatment of nonground deformation processes during the inversion, to utilize more robust estimation algorithms, and to further constrain ground deformation by incorporating ground-based geodetic measurements. In this section, we outline several issues that need to be addressed in future revisions of MInTS.

[45] The method described here can be easily extended to include multiple viewing geometries (i.e., ascending and descending orbits, or other satellite platforms). With sufficient observations on multiple viewing geometries, there is the potential to recover the underlying $3 \mathrm{D}$ deformation. Additionally, the wavelet based approach may possibly be extended to operate on the original wrapped interferograms, thereby avoiding errors incurred in unwrapping [e.g., Goldstein et al., 1988; Chen and Zebker, 2001; Simons and Rosen, 2007].

[46] In our representation of interferometric measurements, equation (10), we lumped all interferometric signal that is not associated with ground deformation into an undescribed noise. Either correcting the interferograms for significant spatially coherent noise prior to MInTS analysis, or simultaneously estimating a model of such noise, may yield a better estimation of ground deformation [e.g., Pritchard et al., 2006; Cavelié et al., 2007; Lin et al., 2010; Shirzaei and Walter, 2011]. Given even a purely horizontally stratified troposphere, one expects topographically correlated delays [e.g., Delacourt et al., 1998; Cavalié et al., 2007]. These so-called "tropostatic delays" are typically a large contribution to the interferometric measurement, and are relatively straightforward to model [e.g., Cavelié et al., 2007; Lin et al., 2010]. Excursions from horizontal stratification, due to atmospheric turbulence are often important [e.g., Lin et al., 2010], and are more difficult to model. In the case of significant turbulent tropospheric signal, high resolution weather models may be used to estimate the spatial variation in wet delay [Foster et al., 2006; Puyssegur et al., 2007].

[47] The interferometric measurements for Long Valley show strong topographically correlated signal (see auxiliary material). Lin et al. [2010] developed a robust method for the estimation of topographically correlated signal using a multiscale decomposition filter-bank approach, and estimated the tropostatic signal in the ERS interferometric data for Long Valley. The multiscale filter-bank approach of Lin et al. [2010] is similar to as is used in MInTS, although their filter-bank is built with Gaussian filters and the estimation of the tropostatic signal is done in the spatial domain. In contrast, MInTS is based on a wavelet-based filter-bank, and the estimation is done in the wavelet domain. The multiscale approach of Lin et al. [2010] may be extended to the use of a wavelet-based filter-bank and the estimation of tropostatic signal in the wavelet domain. In which case, it would be trivial to incorporate the simultaneous estimation of tropostatic signal and ground-deformation into MInTS.

[48] In the regularized estimation, we use an $n$-fold crosscorrelation to select the optimal penalty parameter. In crossvalidation, it is essential that the data in the learning and testing sets are independent [Picard and Cook, 1984]. In the case of inverting the wavelet coefficients of InSAR interferograms, ensuring the independence of the learning and testing sets is only possible if the interferograms in the learning and testing sets share no common SAR acquisitions. Hence, to ensure that the interferograms in the learning and testing are formed from different radar acquisitions it is likely that interferograms would need to be held out of both the learning and testing sets. The particular interferograms that would need to be removed would depend on the $n$ random learning/testing sets in the $n$-fold crossvalidation. The random removal of data from the estimation scheme is problematic. For instance, particular folds in which a larger number of interferograms are dropped might have significantly less resolving power that other folds in the cross-validation, thereby complicating the selection of the optimal penalty parameter [e.g., Picard and Cook, 1984]. We have tested the cross-validation scheme using learning/ testing sets formed on the interferograms and on the acquisitions, and found that in almost all instances the crossvalidation returns the lowest tested $\lambda$ (i.e., noisiest model) when the data are partitioned based on interferograms. When the learning/testing sets are formed on SAR acquisitions, the cross-validation is almost always stable, with a clear $\lambda$ in which the estimated models from inverting all learning sets best fits the data in the associated testing sets. When the learning/testing sets are formed from the acquisitions, as long as the number of folds in the cross-validation is not comparable to the number of acquisitions, the dependence between the learning and testing sets is minimized. In MInTS, cross-validation is not required, and users can choose to invert for ground deformation with specified penalty parameters.

[49] The estimation of ground deformation in the present version of MInTS is entirely built upon least squares. The use of a purely least squares approach, relying on an L2 norm, is somewhat debatable since phase noise is not Gaussian. Additionally, interferometric noise contains other non-Gaussian processes, such as unwrapping errors [e.g., Goldstein et al., 1988; Chen and Zebker, 2001], and tropospheric path delays [e.g., Massonnet et al., 1993; Goldstein, 1995]. It may be more robust to use an L1 norm when dealing with interferometric data.

[50] Due to the often large contributions to interferometric measurements from processes other than ground deformation, it is difficult to constrain ground deformation during periods of low deformation rates. This can be readily seen in our demonstration of MInTS with the Long Valley InSAR stack (Figures 7-8), where although the deformation we estimated is broadly consistent with ground-based geodetic measurements, our estimation does not capture the small amplitude deformation after about 2002. It may be that the Envisat interferograms are dominated by topographically correlated signal, and simultaneous estimation of a tropostatic noise model may improve the estimates of the spatiotemporal 
ground deformation. However, our inability to capture the low signal deformation may also be due to the inherent sensitivity of interferometric measurements. Incorporation of ground based deformation measurements, primarily GPS, into the MInTS analysis has the potential to add strong constraints on the spatiotemporal ground deformation, as ground based geodetic measurements tend to include much more redundant time sampling than InSAR measurements. GPS and InSAR are largely complementary measurements, as GPS is a more continuous record of time-dependent ground deformation at specific locations, while InSAR provides a near continuous image of ground offsets. Incorporating the point-measurements of GPS into the multiscale wavelet decomposition of MInTS is not a straightforward task, and we save it for a future study.

\section{Conclusions}

[51] We present MInTS, a new approach to extracting continuous spatiotemporal ground deformation from InSAR catalogs. We use a Meyer wavelet decomposition, which is chosen in order to efficiently deal with the spatial covariances in the InSAR phase difference measurements, as the Meyer wavelet transform essentially diagonalizes the interferogram spatial covariances. We base MInTS on a generalized model of time-dependent ground deformation, that can include linear rates, offsets, logarithmic or exponential trends, sinusoidal oscillations, and a collection of B-splinebased basis functions. We propose the B-spline-based representation of time-dependent ground deformation to capture unknown processes, while the other functions may be used to parameterize known processes (e.g., earthquakes, postseismic deformation, seasonal signals).

[52] The estimation of ground deformation is done in the wavelet-domain at each location and scale independently. We use a regularized least squares estimation, optionally using $n$-fold cross-validation in order to select the optimal regularization penalty parameter. We include an additional model-resolution-based regularization in the estimation, which may be used to more heavily damp the model during periods of sparse SAR acquisitions, compared to during periods of dense acquisitions. The MInTS approach provides an efficient method for interpolating across regions of low interferometric correlation, providing a constrained estimation of the continuous spatiotemporal ground deformation field. In this paper, we only focus on unwrapped interferograms from a single viewing geometry, so this approach only resolves ground deformation along the satellite lineof-sight. MInTS is developed as a toolbox in Matlab, and provides an extensible framework for estimation of deformation from catalogs of interferograms.

[53] We illustrate the application of MInTS to a catalog of 92 ERS and Envisat interferograms in the Long Valley, CA, region, spanning 16 years. The MInTS estimation of ground deformation resolves the uplift in the caldera prior to 1999 , with accelerated uplift initiating in late 1998. In early 1999, the ground slightly subsides within the caldera, and then slightly inflates again prior to 2004. Following 2004, there is a larger subsidence signal over the western regions of the Long Valley caldera, with more pronounced subsidence localized at the Casa Diablo geothermal plant. The estimated deformation is broadly consistent with that detected by ground-based geodetic measurements and conventional InSAR time series techniques.

\section{Appendix A: Decorrelating Power of Orthogonal Wavelet Bases}

[54] In this appendix we review classical properties of the covariance structure of wavelet coefficients, discuss the intra and inter-scale covariances of the wavelet coefficients, and demonstrate the decorrelating power of the orthogonal wavelet transform. For the sake of simplicity, this analysis is presented for one dimensional stochastic processes, whereas interferograms are $2 \mathrm{D}$.

[55] We define $\mathbb{E}[X]$ to be the expectation of $X$ and $\operatorname{Cov}[X, Y]$ to be the covariance between $X$ and $Y$. For zero mean processes, $\operatorname{Cov}[X, Y]=\mathbb{E}[X Y]$. A stochastic process $\{X(t), t \in \mathbb{R}\}$ is said to be wide sense stationary (WSS) if $\mathbb{E}\left[|X(t)|^{2}\right]<\infty, \forall t \in \mathbb{R}, \mathbb{E}[X(t)]=\mu$, and $\forall r \in \mathbb{R}, \operatorname{Cov}[X(t)$, $X(t+r)]=f(r)$. For an orthonormal wavelet basis of $L^{2}(\mathbb{R})$,

$$
\left\{\psi_{j, n}(t)=2^{-j / 2} \psi\left(2^{-j} t-n\right)\right\}_{(j, n) \in \mathbb{Z}^{2}},
$$

where $\psi$ is the mother wavelet, and we denote the wavelet coefficients of $f$ at scale $j$ and location $n$ by $d_{j, n}=\left\langle f, \psi_{j, n}\right\rangle$. We are interested in characterizing the covariance structure of the wavelet coefficients of a WSS process with given autocovariance function $f$. Note that since the wavelets have zero mean and $\mathbb{E}[X(t)]$ is constant, the wavelet coefficients of $f$ also have zero mean. Hence, the covariance of $d_{j, n}$ and $d_{k, m}$ is just the autocorrelation:

$$
\begin{aligned}
\mathbb{E}\left[d_{j, n} d_{k, m}\right] & =\mathbb{E}\left[\int_{\mathbb{R}} \psi_{j, n}(t) X(t) d t \int_{\mathbb{R}} \psi_{k, m}(s) X(s) d s\right] \\
& =\int_{\mathbb{R}} \psi_{j, n}(t)\left(\int_{\mathbb{R}} \mathbb{E}[X(t) X(s)] \psi_{k, m}(s) d s\right) d t \\
& =\int_{\mathbb{R}} \psi_{j, n}(t)\left(\int_{\mathbb{R}} f(t-s) \psi_{k, m}(s) d s\right) d t \\
& =\int_{\mathbb{R}} \psi_{j, n}(t)\left(f * \psi_{k, m}(t)\right) d t .
\end{aligned}
$$

Introducing the function $\bar{\psi}_{j, n}(t)=\psi_{j, n}(-t)$, the last equation can be written as a double convolution:

$$
\begin{aligned}
\mathbb{E}\left[d_{j, n} d_{k, m}\right] & =\int_{\mathbb{R}} \bar{\psi}_{j, n}(0-t)\left(f * \psi_{k, m}(t)\right) d t \\
& =\bar{\psi}_{j, n} * f * \psi_{k, m}(0) .
\end{aligned}
$$

Our analysis can be simplified by writing equation (A7) in terms of the Fourier transforms of the involved functions, by means of the inverse Fourier transform evaluated at $t=0$ :

$$
\mathbb{E}\left[d_{j, n} d_{k, m}\right]=\frac{1}{2 \pi} \int_{\mathbb{R}} \widehat{f}(\omega) \widehat{\psi}_{j, n}^{*}(\omega) \widehat{\psi}_{k, m}(\omega) d \omega
$$

where we denote the Fourier transform of $\psi$ as $\widehat{\psi}$, and $\widehat{\psi} *$ as the complex conjugate of $\widehat{\psi}(\widehat{\psi} *$ is the Fourier transform of $\bar{\psi})$. 


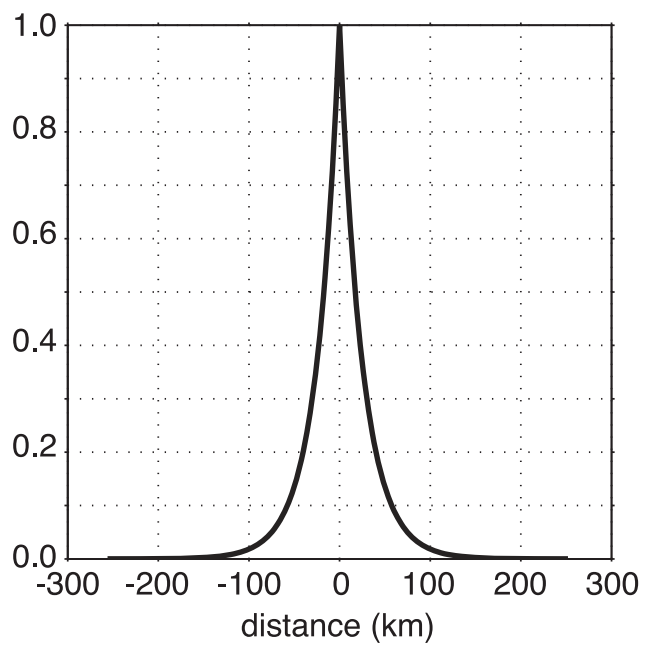

Figure A1. Exponential spatial domain noise covariance function, which is the 1D equivalent of equation (12).

Then, replacing the wavelets by its expressions in terms of the mother wavelet, $\psi$, we find

$\mathbb{E}\left[d_{j, n} d_{k, m}\right]=\frac{\sqrt{2^{(j+k)}}}{2 \pi} \int_{\mathbb{R}} \widehat{f}(\omega) \widehat{\psi}^{*}\left(2^{j} \omega\right) \widehat{\psi}\left(2^{k} \omega\right) \exp \left(i \omega\left(2^{j} n-2^{k} m\right)\right) d \omega$.

[56] In the following we assume the wavelets have compact spectral support. Introducing the positive constant $A$, we denote the spectral support of the mother wavelet by $\operatorname{supp}(\widehat{\psi})=[-2 A-\delta,-A] \cup[A, 2 A+\delta]$, and we assume that $0<\delta<2 A$, which is a totally non-restrictive condition for orthogonal wavelet bases.

[57] Let us assume without loss of generality that $k=j+l$, with $l \geq 0$. Then

$$
\begin{aligned}
\left|\mathbb{E}\left[d_{j, n} d_{j+l, m}\right]\right| & \leq \frac{2^{(j+l / 2)}}{2 \pi} \int_{\mathbb{R}}|\widehat{f}(\omega)|\left|\widehat{\psi} *\left(2^{j} \omega\right)\right|\left|\widehat{\psi}\left(2^{j+l} \omega\right)\right| d \omega \\
& \leq \frac{2^{(j+l / 2)}}{\pi} \int_{2^{-j} A}^{2^{-(j+l-1)} A+2^{-(j+l)} \delta}|\widehat{f}(\omega)| d \omega .
\end{aligned}
$$

a)

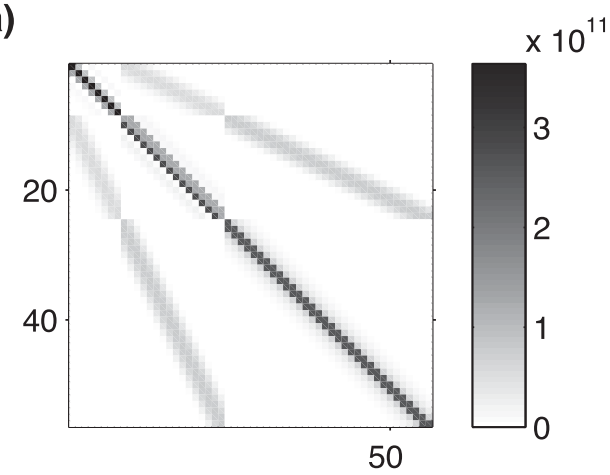

Since $\delta<2 A$, it follows that

$$
\left|\mathbb{E}\left[d_{j, n} d_{j+l, m}\right]\right| \leq \frac{2^{(j+l / 2)}}{\pi} \int_{2^{-j} A}^{2^{-(j+l-2)} A}|\widehat{f}(\omega)| d \omega .
$$

Consequently, $\forall l \geq 2, \quad \mathbb{E}\left[d_{j, n} d_{j+l, m}\right]=0$, which is to say that all wavelet coefficients separated by at least two scales are uncorrelated. For two neighboring scales (i.e., $l=1$ ), it follows from (A10) that

$$
\left|\mathbb{E}\left[d_{j, n} d_{j+1, m}\right]\right| \leq \frac{\delta}{\pi \sqrt{2}} \max \left\{|\widehat{f}(\omega)|: 2^{-j} A \leq \omega \leq 2^{-j} A+2^{-(j+1)} \delta\right\} .
$$

Finally, for wavelet coefficients at the same scale, $j=k$ in equation (A9), and the covariance of intrascale wavelet coefficients is given by

$$
\begin{aligned}
\mathbb{E}\left[d_{j, n} d_{j, m}\right] & =\frac{2^{j}}{2 \pi} \int_{\mathbb{R}} \widehat{f}(\omega)\left|\widehat{\psi}\left(2^{j} \omega\right)\right|^{2} \exp \left(2^{j} i \omega(n-m)\right) d \omega \\
& =\frac{1}{2 \pi} \int_{\mathbb{R}} \widehat{f}\left(2^{-j} \xi\right)|\widehat{\psi}(\xi)|^{2} \exp (i \xi(n-m)) d \xi \\
& =: g(n-m) .
\end{aligned}
$$

Hence, if $\widehat{f}$ and $\widehat{\psi}$ are both of class $C^{p}, g(n-m)$ will decay as $1 /|n-m|^{p+1}$. By choosing, for instance, Meyer wavelets with $\delta \rightarrow 0$ (for any $\delta>0$, Meyer wavelets are $C^{\infty}$ in the spectral domain), one can still have $|\widehat{\psi}|^{2} \in C^{\infty}[$ Daubechies, 1992]. In this case, the covariance between neighboring scales can be made arbitrarily small, and the decay of the intrascale coefficients will be given by the regularity of $\widehat{f}$.

[58] We consider two test functions, a delta distribution and an exponential spatial covariance function (Figure A1). The latter is a 1D version of the spatial covariance in equation (12) and from Emardson et al. [2003]. For both cases, we show the wavelet covariance matrices for the Meyer wavelets. The delta function is used to illustrate the case where the coupling between different scales or locations is the highest possible. This can be readily seen from equation (A10) and (A15), since the Fourier Transform of the delta distribution is a constant, the decay in the covariance with scales or locations depends only on the wavelet

b)

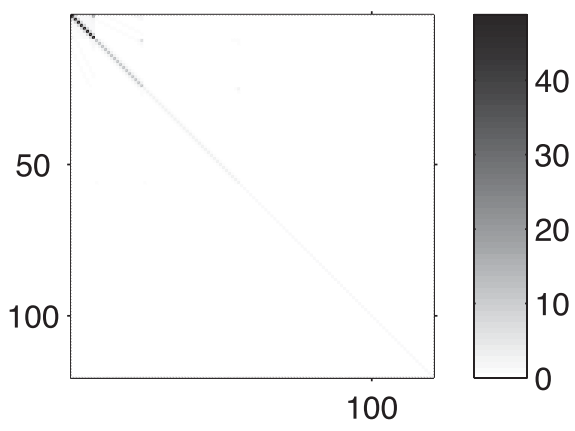

Figure A2. (a) Absolute value of the covariance matrix of Meyer wavelet coefficients assuming a delta distribution spatial covariance function. (b) Absolute value of the covariance matrix of Meyer wavelet coefficients assuming the covariance function in Figure A1. 
spectrum. The second test covariance function is similar to those from nearly signal free interferograms [e.g., Lohman and Simons, 2005]. For the delta distribution, the wavelet transform of the covariance has non-trivial off-diagonal elements (Figure A2a), indicating covariances both between wavelet coefficients at the same scale and in different scales. In the case of the exponential spatial covariance function, most of the off-diagonal terms are small, indicating negligible correlation of the wavelet coefficients across scales (Figure A2b).

[59] Acknowledgments. We thank the editor, Tom Parsons, and two anonymous reviewers. This work was partially supported by NASA grant NNX09AD25G to M.S.

\section{References}

Aster, R. C., B. Borchers, and C. H. Thurber (2005), Parameter Estimation and Inverse Problems, Elsevier Acad., Burlington, Mass.

Bennett, R. A. (2008), Instantaneous deformation from continuous GPS: Contributions from quasi-periodic loads, Geophys. J. Int., 174, 1052-1064, doi:10.1111/j.1365-246X.2008.03846.x.

Berardino, P., G. Fornaro, R. Lanari, and E. Sansosti (2002), A new algorithm for surface deformation monitoring based on small baseline differential SAR interferograms, IEEE Trans. Geosci. Remote Sens., 40, 2375-2383.

Bondár, I., and K. McLaughlin (2009), Seismic location bias and uncertainty in the pressence of correlated and non-Gaussian travel-time errors, Bull. Seismol. Soc. Am., 99, 172-193.

Buckheit, J. B., and D. L. Donoho (1995), WaveLab and reproducible research, report, 27 pp., Dep. of Stat., Stanford Univ., Stanford, Calif. (Available at http://www-stat.stanford.edu/donoho/Reports/1995/wavelab.pdf.)

Bürgmann, R., G. Hilley, A. Ferretti, and F. Novali (2006), Resolving vertical tectonics in the San Francisco Bay Area from permanent scatterer InSAR and GPS analysis, Geology, 34, 221-224.

Cavalié, O., M. P. Doin, C. Lasserre, and P. Briole (2007), Ground motion measurement in the Lake Mead area, Nevada, by differential synthetic aperture radar interferometry time series analysis: Probing the lithosphere rheological structure, J. Geophys. Res., 112, B03403, doi:10.1029/ 2006JB004344

Chen, C. W., and H. A. Zebker (2001), Two-dimensional phase unwrapping with use of statistical models for cost functions in nonlinear optimization, J. Opt. Soc. Am. A, 18, 338-351.

Colesanti, C., A. Ferretti, F. Novali, C. Prati, and F. Rocca (2003), SAR Monitoring of progressive and seasonal ground deformation using the permanent scatterers technique, IEEE Trans. Geosci. Remote Sens., 41, $1685-1701$

Daubechies, I. (1992), Ten Lectures on Wavelets, 357 pp., Soc. for Indust. and Appl. Math., Philadelphia, Pa.

Delacourt, C., P. Briole, and J. Achache (1998), Tropospheric corrections of SAR interferograms with strong topography: Application to Etna, Geophys. Res. Lett., 25, 2849-2852.

Dong, D., T. A. Herring, and R. W. King (1998), Estimating regional deformation from a combination of space and terrestrial geodetic data J. Geodesy, 72, 200-214

Dong, D., P. Fang, Y. Bock, M. K. Cheng, and S. Miyazaki (2002), Anatomy of apparent seasonal variations from GPS-derived site position time series, J. Geophys. Res., 107(B4), 2075, doi:10.1029/2001JB000573.

Doubre, C., and G. Peltzer (2007), Fluid-controlled faulting process in the Asal Rift, Djibouti, from 8 yr of radar interferometry observations, Geology, 35, 69-72.

Emardson, T. R., M. Simons, and F. H. Webb (2003), Neutral atmospheric delay in interferometric synthetic aperture radar applications: Statistical description and mitigation, J. Geophys. Res., 108(B5), 2231, doi:10.1029/ 2002JB001781.

Feng, L., and A. V. Newman (2009), Constraints on continued episodic inflation at Long Valley Caldera, based on seismic and geodetic observations, J. Geophys. Res., 114, B06403, doi:10.1029/2008JB006240.

Ferretti, A., C. Prati, and F. Rocca (2000), Nonlinear subsidence rate estimation using permanent scatterers in differential SAR interferometry, IEEE Trans. Geosci. Remote Sens., 38, 2202-2212.

Ferretti, A., C. Prati, and F. Rocca (2001), Permanent scatterers in SAR interferometry, IEEE Trans. Geosci. Remote Sens., 39, 8-20.

Foster, J., B. Brooks, T. Cherubini, C. Shacat, S. Businger, and C. L. Werner (2006), Mitigating atmospheric noise for InSAR using a high resolution weather model, Geophys. Res. Lett., 33, L16304, doi:10.1029/2006GL026781.
Geisser, S. (1975), The predictive sample resuse method with applications, J. Am. Stat. Assoc., 70, 320-328.

Goldstein, R. M. (1995), Atmospheric limitations to repeat-track radar interferometry, Geophys. Res. Lett., 22, 2517-2520.

Goldstein, R. M., H. A. Zebker, and C. L. Werner (1988), Satellite radar interferometry: Two-dimensional phase unwrapping, Radio Sci., 23, 713-720.

Golub, G. H., and C. F. van Loan (1980), An analysis of the total least squares problem, SIAM J. Numer. Anal., 17, 883-893.

Gourmelen, N., and F. Amelung (2005), Post-seismic mantle relaxation in the Central Nevada Seismic Belt, Science, 310, 1473-1476.

Hansen, P. C. (1994), A Matlab package for analysis and solution of discrete ill-posed problems, Numer. Algorithms, 6, 1-35.

Hansen, P. C. (2007), Regularization tools version 4.0 for Matlab 7.3, Numer. Algorithms, 46, 184-194.

Hanssen, R. F. (2001), Radar Interferometry: Data Interpretation and Error Analysis, 308 pp., Kluwer, Dordrecht, Netherlands.

Herring, T. A., J. L. Davis, and I. I. Shapiro (1990), Geodesy by radio interferometry: The application of Kalman filtering to the analysis of very long baseline interferometry data, J. Geophys. Res., 95, 12,561-12,581.

Hetland, E. A., and B. H. Hager (2006), The effects of rheological layering on postseismic and interseismic displacements, Geophys. J. Int., 166 , 272-292, doi:10.1111/j.1365-246X.2006.02974.x.

Hooper, A. (2008), A multi-temporal InSAR method incoperating both persistent scatterrer and small baseline approaches, Geophys. Res. Lett., 35, L16302, doi:10.1029/2008GL034654.

Hooper, A., H. Zebker, P. Segall, and B. Kampes (2004), A new method for measuring deformation on volcanoes and other natural terrains using InSAR persistent scatterers, Geophys. Res. Lett., 31, L23611, doi:10.1029/2004GL021737.

Howle, J. F., J. O. Langbein, C. D. Farrarr, and S. K. Wilkinson (2003), Deformation near the Casa Diablo geothermal well field and related processes Long Valley caldera, Eastern California, 1993-2000, J. Geotherm. Res., 127, 365-390.

Knospe, S. H.-G., and S. Jònsson (2010), Covariance estimation for dInSAR surface deformation measurements in the pressence of anisotropic atmospheric noise, IEEE Trans. Geosci. Remote Sens., 48, 2057-2065.

Krzanowski, W. J., P. Jonathan, W. V. McCarthy, and M. R. Thomas (1995), Analysis with singular covariance matricies: Methods and applications to spectroscopic data, J. R. Stat. Soc., Ser. C, 44, 101-115.

Lanari, R., O. Mora, M. Manunta, J. J. Mallorqui, P. Berardino, and E. Sansosti (2004), A small baseline approach for investigating deformations on full resolution differential SAR interferograms, IEEE Trans. Geosci. Remote Sens., 42, 1377-1386.

Langbein, J. O. (2003), Deformation of the Long Valley Caldera, California: Inferenced from measurements from 1988 to 2001, J. Volcanol. Geotherm. Res., 127, 247-267.

Langbein, J. O., D. P. Hill, T. N. Parker, and S. K. Wilkinson (1993), An episode of re-inflation of the Long Valley caldera, eastern California, 1989-1991, J. Geophys. Res., 98, 15,581-15,870.

Langbein, J. O., D. Dzurisin, G. Marshall, R. Stein, and J. Rundle (1995), Shallow and peripheral volcanic sources of inflation revealed by modeling two-color geodimeter and leveling data from Long Valley caldera, California, 1988-1992, J. Geophys. Res., 100, 12,487-12,495.

Lin, Y.-N., M. Simons, E. A. Hetland, P. Musè, and C. DiCaprio (2010), A multi-scale approach to estimating topographically correlated propegation delays in radar interferometry, Geochem. Geophys. Geosyst., 11, Q09002, doi:10.1029/2010GC003228.

Lohman, R. B. (2004), The inversion of geodetic data for earthquake parameters, $\mathrm{PhD}$ thesis, Calif. Inst. of Technol., Pasadena.

Lohman, R. B., and M. Simons (2005), Some thoughts on the use of InSAR data to constrain models of surface deformation, Geochem. Geophys. Geosyst., 6, Q01007, doi:10.1029/2004GC000841.

Lundgren, P., S. Usai, E. Sansosti, R. Lanari, M. Tesauro, G. Fornaro, and P. Berardino (2001), Modeling surface deformation observed with synthetic aperture radar interferometry at Campi Flegrei caldera, J. Geophys. Res., 106, 19,355-19,366.

Lundgren, P., E. A. Hetland, Z. Liu, and E. F. Fielding (2009), Southern San Andreas-San Jacinto fault system slip rates estimated from earthquake cycle models constrained by GPS and InSAR observations, J. Geophys. Res., 114, B02403, doi:10.1029/2008JB005996.

Lyons, S., and D. Sandwell (2003), Fault creep along the southern San Andreas from interferometric synthetic aperture radar, permanent scatterers, and stacking, J. Geophys. Res., 108(B1), 2047, doi:10.1029/ 2002JB001831.

Mallat, S. (2008), A Wavelet Tour of Signal Processing: The Sparse Way, 3rd ed., 832 pp., Academic, Burlington, Mass. 
Massonnet, D., M. Rossi, C. Carmona, F. Adragna, G. Peltzer, K. Fiegl, and T. Rabaute (1993), The displacement field of the Landers earthquake mapped by radar interferometry, Nature, 364, 138-142.

Peltzer, G., F. Crampe, S. Hensley, and P. Rosen (2001), Transient strain accumulation and fault interaction in the Eastern California Shear Zone, Geology, 29, 975-978.

Picard, R. R., and R. D. Cook (1984), Cross-validation of regression models, J. Am. Stat. Assoc., 79, 575-583.

Pritchard, M. E., and M. Simons (2006), An aseismic slip pulse in northern Chile and along-strike variations in seismogenic behavior, J. Geophys. Res., 111, B08405, doi:10.1029/2006JB004258.

Pritchard, M. E., C. Ji, and M. Simons (2006), Distribution of slip from $11 M_{w}>6$ earthquakes in the northern Chile subduction zone, J. Geophys. Res., 111, B10302, doi:10.1029/2005JB004013.

Puyssegur, B., R. Michel, and J. P. Avouac (2007), Tropospheric phase delay in interferometric synthetic aperture radar estimated from meteorological model and multispectral imagery, J. Geophys. Res., 112, B05419, doi:10.1029/2006JB004352.

Savage, J. C., J. L. Svarc, and S. B. Yu (2005), Postseismic relaxation and transient creep, J. Geophys. Res., 110, B11402, doi:10.1029/2005JB003687.

Savage, J. M., J. L. Svarc, and W. H. Prescott (2003), Near-field postseismic deformation associated with the 1992 Landers and 1999 Hector Mine, California, earthquakes, J. Geophys. Res., 108(B9), 2432, doi:10.1029/ 2002JB002330.

Schmidt, D. A., and R. Bürgmann (2003), Time-dependent land uplift and subsidence in the Santa Clara valley, California, from a large interferometric synthetic aperture radar data set, J. Geophys. Res., 108(B9), 2416, doi:10.1029/2002JB002267.

Shirzaei, M., and T. R. Walter (2011), Estimating the effect of satellite orbital error using wavelet-based robust regression applied to InSAR deformation data, IEEE Trans. Geosci. Remote Sens., 49, 4600-4605, doi:10.1109/TGRS.2011.2143419.
Simons, M., and P. A. Rosen (2007), Interferometric synthetic aperture radar geodesy, in Treatise on Geophysics, vol. 3 Geodesy, edited by G. Schubert, pp. 391-447, Elsevier, Amaterdam.

Simons, M., Y. Fialko, and L. Rivera (2002), Coseismic deformation from the $1999 M_{w} 7.1$ Hector Mine, California, earthquake as inferred from InSAR and GPS observations, Bull. Seismol. Soc. Am., 92, 1390-1402.

Stone, M. (1974), Cross-validatory choice and assessment of statistical predictions, J. R. Stat. Soc., Ser. B, 36, 111-147.

Tizzani, P., B. Berardino, F. Casu, P. Euillades, M. Manzo, G. P. Ricciardi, G. Zeni, and R. Lanari (2007), Surface deformation of Long Valley caldera and Mono Basin, California, investigated with the SBAS-InSAR approach, Remote Sens. Environ., 108, 277-289.

Tizzani, P., M. Battaglia, G. Zeni, S. Atzori, P. Berardino, and R. Lanari (2009), Uplift and magma intrusion at Long Valley caldera from InSAR and gravity measurements, Geology, 37, 63-66.

Wegmüller, U. (2003), Potential of interferometry point target analysis using small data stacks, paper presented at Fringe '03 Workshop, Eur. Space Agency, Frascati, Italy.

Whitney, H. (1932), Non-separable and planar graphs, Trans. Am. Math. Soc., 34, 339-362.

P. S. Agram, Y. N. Lin, and M. Simons, Seismological Laboratory, California Institute of Technology, MC252-21, 1200 E. California Blvd., Pasadena, CA 91125, USA.

C. J. DiCaprio, ExxonMobil Upstream Research Co., 3120 Buffalo Spdwy., Houston, TX 77098, USA.

E. A. Hetland, Department of Earth and Environmental Sciences, University of Michigan, 1100 N. University Ave., Ann Arbor, MI 48109 USA. (ehetland@alum.mit.edu)

P. Musé, Department of Signal and Image Processing, IIE, Facultad de Ingenieria, Universidad de la Republica, 11300 Montevideo, Uruguay. 